\title{
CHARACTERISTIC CLASSES OF REAL MANIFOLDS IMMERSED IN COMPLEX MANIFOLDS( $\left.{ }^{1}\right)$
}

\author{
BY \\ HON-FEI LAI
}

\begin{abstract}
Let $M$ be a compact, orientable, $k$-dimensional real differentiaable manifold and $N$ an $n$-dimensional complex manifold, where $k \geq n$. Given an immersion $\iota: M \rightarrow N$, a point $x \in M$ is called an RC-singular point of the immersion if the tangent space to $\iota(M)$ at $\iota(x)$ contains a complex subspace of dimension $>k-n$. This paper is devoted to the study of the cohomological properties of the set of RC-singular points of an immersion.

When $k=2 n-2$, the following formula is obtained:
\end{abstract}

$$
\Omega(M)+\sum_{r=0}^{n-1} \widetilde{\Omega}(\iota)^{n-r-1} \iota^{*} c_{r}(N)=2 t^{*} D K,
$$

where $\Omega(M)$ is the Euler class of $M, \widetilde{\Omega}(\iota)$ is the Euler class of the normal bundle of the immersion, $c_{r}(N)$ are the Chern classes of $N$, and $t^{*} D K$ is a cohomology class of degree $2 n-2$ in $M$ whose value on the fundamental class of $M$ gives the algebraic number of RC-singular points of $\iota$. Various applications are discussed.

For $n \leq k<2 n-2$, it is shown that, as long as dimensions allow, all Pontrjagin classes and the Euler class of $M$ are carried by subsets of the set of RC-singularities of an immersion $\iota: M \rightarrow C^{n}$.

1. Introduction. The differential geometry of real submanifolds of differentiable manifolds and complex subvarieties of complex manifolds have been studied extensively for a long time, but the differential geometry of real submanifolds of complex manifolds has not been explored to any great extent. Among the earliest works in this area is a paper of E. Cartan [2], where he studied the pseudoconformal geometry of hypersurfaces in two-dimensional complex Euclidean space, that is, he studied the invariants of hypersurfaces when the ambient complex space is subject to holomorphic transformations. He also classified the hypersurfaces both locally and globally according to pseudoconformality. The main feature of the paper is the method of equivalence that he developed earlier.

Received by the editors November 12, 1971.

AMS 1970 subject classifications. Primary 53C99, 57D20; Secondary 55F40, 32-00.

Key words and phrases. Complex manifold, real submanifold, Poincaré duality, intersection, Schubert varieties, vector bundles, characteristic classes, cohomological extension of fibres.

(1) This paper forms part of the dissertation of the author at the University of California, Berkeley. 
Using calculations with some special Lie groups and Lie algebras, N. Tanaka [11] generalized Cartan's results to hypersurfaces in higher dimensional complex Euclidean spaces. He attached to each hypersurface a canonical fibre bundle and connection which together characterize the pseudoconformal equivalence class of the hypersurface, under some nondegeneracy assumptions. He also studied the group of pseudoconformal transformations of such hypersurfaces. With more refined techniques, namely generalized graded Lie algebras, he was able [12] to extract some information on the dimension of the group of pseudoconformal transformations of a real submanifold of a complex Euclidean space, for general codimensions.

The real submanifolds studied by Cartan and Tanaka are those which satisfy a certain condition of regularity, namely that the maximal complex subspace of the tangent space to the real submanifold has the minimal dimension. More precisely, if $\iota: M^{(k)} \rightarrow \mathbf{C}^{n}$ is an immersion of a $k$-dimensional differentiable manifold in an $n$-dimensional complex Euclidean space with $n \leq k$, then

$$
\operatorname{dim}_{C} \iota_{*} T_{x} \cap J \iota_{*} T_{x} \geq k-n
$$

for all $x \in M$, where $T_{x}$ is the tangent space to $M$ at $x$, and $J$ is the almost complex structure in $\mathbf{C}^{n}$. A point $x \in M$ is called an RC-singular point if

$$
\operatorname{dim}_{\mathbf{C}} \iota_{*} T_{\boldsymbol{x}} \cap J \iota_{*} T_{\boldsymbol{x}}>k-n
$$

otherwise it is called an RC-regular point. Then the works of Cartan and Tanaka are concerned with real submanifolds with no $\mathbf{R C}$-singular points.

RC-singular points have been studied by analysts under the name of "nongeneric points" (see for example the papers of Wells in the Bibliography). The extendibility of holomorphic functions on real submanifolds depends very much on RC-regularity. For example, there is a theorem of Wells [14] which states that a compact manifold of dimension $k$ immersed without RC-singular points in a complex Euclidean space of dimension $n<k$ is extendible to a manifold of one higher dimension. Also, at an RC-regular point, the vanishing or nonvanishing of the Levi form is equivalent to local holomorphicity or local extendibility of the real submanifold.

The significance of RC-regularity thus deserves its deeper study, and in this connection Wells [15] has derived topological restrictions on a compact orientable manifold for it to be embeddable without RC-singular points in some Euclidean space. Namely, the Euler class and all the Pontrjagin classes must vanish. It is therefore natural to expect that these characteristic classes of the manifold are somehow carried by the set of RC-singular points of a general immersion. To show that this is indeed the case is the main purpose of this paper. 
In $\$ 2$ we explain the terminology "RC-singularity" and the basic notations, and show that the set of RC-singular points can be smoothed out to form a submanifold by merely deforming the immersion.

$\$ 3$ reviews some known facts about products in cell complexes, about Schubert varieties in Grassmannian manifolds, and about characteristic classes of vector bundles. These are the main tools for the rest of the paper.

In $\$ 4$ we find a canonical basis for the cohomology of the Grassmannian manifold of oriented $2 m$-planes in $(2 m+2)$-dimensional Euclidean space, and the main theorem of the section, Theorem 4.8, shows how the interaction between the real and complex structures of a Euclidean space enters into the cohomology ring structure of the Grassmannian manifold. Some applications are drawn to immersions in complex Euclidean spaces.

$\$ 5$ gives the generalization to immersions of $2 m$-dimensional real manifolds in $(m+1)$-dimensional complex manifolds. The main theorem, Theorem 5.10, gives a formula relating all the real and complex characteristic classes of the manifolds in question with the set of RC-singular points of the immersion. Particular cases of this formula include the duality theorems for Stiefel-Whitney classes and Chern classes.

For immersions in higher codimensions, we see in $\$ 6$ that the Euler class and dual Pontrjagin classes of a real manifold are always carried by the set of RC-singular points of an immersion of the manifold into a complex Euclidean space of the appropriate dimension. The results are summarized in $\$ 7$ by a statement on the size of the ring of cohomology classes carried by the set of RC-singular points. .

In the last section, second order RC-singular points are considered and a simple case is described.

The author wishes to thank Professor S. S. Chern for his continual advice and encouragement during the whole research.

2. Generic immersions. Throughout this paper, $M^{(k)}$ will always denote a compact, orientable, $k$-dimensional differentiable $\left(C^{\infty}\right)$ manifold, and $N_{(n)}$ will denote an $n$-dimensional complex manifold.

Let $\iota: M^{(k)} \rightarrow N_{(n)}$ be an immersion of $M$ in a complex manifold of complex dimension $n \leq k$. Then by linear algebra we know that

$$
\operatorname{dim}_{\mathrm{C}} \iota_{*} T_{x}(M) \cap J \iota_{*} T_{x}(M) \geq k-n,
$$

where $T_{x}(M)$ is the tangent space to $M$ at $x, \iota_{*}$ is the induced map of tangent spaces, and $J$ is the almost complex structure in $N$.

Definition 2.1. A point $x \in M$ is called an RC-singular point of the immersion $\iota$ if 


$$
\operatorname{dim}_{C} \iota_{*} T_{x}(M) \cap J \iota_{*} T_{x}(M)>k-n .
$$

Remark on the terminology. The word "singular" is used here, as usual in differential geometry, to signify that a certain matrix fails to have maximal rank at a point, or, equivalently, that a certain real-valued function vanishes at that point. In our case, the matrix is the complex Jacobian matrix in local coordinates (complex-valued function of real variables), and the corresponding real-valued function is the sum of the squares of the absolute values of the $n \times n$ minors of the Jacobian matrix. The prefix RC reminds us of the interaction between the real and complex structures. An RC-singular point has also been called a "nongeneric" point by Wells [14], but we will reserve the adjective "generic" for a familiar topological use.

We now specialize to the case where $N=\mathrm{C}^{n}$, the complex Euclidean space of dimension $n$. Let $\mathbf{R}_{k, l}, l=2 n-k$, be the Grassmannian of oriented $k$-planes (through the origin) in $\mathbf{R}^{k+l}=\mathbf{C}^{n}$. Let $K_{k, l}$ denote the subset of $\mathbf{R}_{k, l}$ consisting of the $k$-planes containing a complex subspace of dimension $k-n+1$ in $\mathbf{C}^{n}$. Then if $t: M \rightarrow \mathbf{R}_{k, l}$ is the Gauss map corresponding to an immersion $\iota: M^{(k)} \rightarrow$ $\mathbf{C}^{n}$, the set of RC-singular points of the immersion is just $t^{-1}\left(K_{k, l}\right)$. We now show that $K_{k, l}$ is an immersed submanifold of $\mathbf{R}_{k, l}$.

Let $\mathrm{C}_{k-n+1}, 2 n-k-1$ be the Grassmannian of complex $(k-n+1)$-planes (through the origin) in $\mathrm{C}^{n}$. Over it is a canonical complex $(2 n-k-1)$-plane bundle whose fibre over a point $X$ is the complex subspace of $\mathrm{C}^{n}$ normal to the complex plane $X$, relative to a fixed complex Euclidean metric in $\mathrm{C}^{n}$. The group of this bundle, $U(2 n-k-1)$, as a subgroup of $S O(4 n-2 k-2)$, acts (nontransitively) on $\mathbf{R}_{2 n-k-2,2 n-k}$ on the left, and so there is an associated bundle with fibre $\mathbf{R}_{2 n-k-2,2 n-k}$. Let $G_{2 n-k-2,2 n-k}\left(\mathbf{C}_{k-n+1,2 n-k-1}\right)$ be its total space. There is a natural map

$$
\psi: G_{2 n-k-2,2 n-k}\left(\mathbf{C}_{k-n+1,2 n-k-1}\right) \rightarrow \mathbf{R}_{k, 2 n-k}
$$

which sends a point $\tilde{X}$ in the fibre over $X \in \mathrm{C}_{k-n+1,2 n-k-1}$ into the oriented $k$-plane in $\mathbf{R}^{2 n}=\mathbf{C}^{n}$ spanned by $X$ and the $(2 n-k-2)$-plane defining $\widetilde{X}$. It is easily seen that $\psi$ is an immersion whose image is $K_{k, 2 n-k}$. From this we also know that

$$
\begin{aligned}
\operatorname{dim} K_{k, 2 n-k} & =(2 n-k-2)(2 n-k)+2(k-n+1)(2 n-k-1) \\
& =k(2 n-k)-2(k-n+1) .
\end{aligned}
$$

In general the set of RC-singular points of an immersion can be very weird, but it can be shown that every immersion can be arbitrarily approximated by a generic one, for which the set of RC-singular points is a submanifold of $M$. First we recall the use of the adjective "generic" by Thom: 
Definition 2.2 (Thom [13]). Let $S$ be a function space which is a Baire space (that is., every nonempty open set is of the second category). A property of functions is called generic if it is owned by all functions belonging to a subset of $S$ whose complement is a nowhere-dense set in $S$.

In the same paper quoted, Thom proves a local lemma which asserts that for a generic map of $\mathbf{R}^{k}$ to $\mathbf{R}^{m}$, the derived map is transversal to any given submanifold of the Euclidean space of the range of the derived map. Using this lemma one easily proves the following theorem:

Theorem 2.3. (i) For a generic immersion (or embedding) of $M^{(k)}$ in $N_{(n)}$ with $k \geq n$, the set of RC-singular points is eitber empty or else forms a submanifold of $M$ of dimension $(2 n-k-2)$. (ii) If $L$ is any immersed submanifold of $\mathbf{R}_{k, 2 n-k}$ of codimension $\leq k$, then for a generic immersion (or embedding) of $\mathrm{M}^{(k)}$ in $\mathrm{C}^{n}$ with $k \geq n$, the inverse image of $L$ under the Gauss map of the immersion is a submanifold of $M$.

This theorem naturally implies that every immersion can be arbitrarily approximated by an immersion whose set of RC-singular points forms a submanifold.

3. Topological preliminaries. The cup and cap products in a compact oriented manifold can be expressed in terms of intersections of homology classes by means of the Poincaré duality. A rather complete account is given in the thesis of Gysin [5]. We summarize the necessary facts here. We will work with integral coefficients unless otherwise stated.

Let $K$ be a finite cell complex. For each dimension $p$, let $x_{i}^{p}, i=1$, $\cdots, n(p)$ be the cells of dimension $p$. Each cell $x_{i}^{p}$ can be considered as a homomorphism on the group of chains by $x_{i}^{p}\left(x_{k}^{p}\right)=\delta_{i k}$, and is therefore a cochain. Let $\sigma\left(x_{i}^{p}, x_{k}^{p-1}\right)$ be the incidence coefficient. Then the boundary and coboundary operators $\partial, \delta$ are defined respectively by the equations

$$
\begin{aligned}
\partial x_{i}^{p} & =\sum_{k=1}^{n(p-1)} \sigma\left(x_{i}^{p}, x_{k}^{p-1}\right) x_{k}^{p-1}, \\
\delta x_{k}^{p-1} & =\sum_{i=1}^{n(p)} \sigma\left(x_{i}^{p}, x_{k}^{p-1}\right) x_{i}^{p} .
\end{aligned}
$$

These give rise to the homology and cohomology groups of $K$.

Now restrict to the case of a compact oriented differentiable manifold $M$ of dimension $k$. Suppose that $M$ is given a cell decomposition $K$ which has a dual cell decomposition $K^{*}$. It is known that any simplicial decomposition of $M$ has a dual cell decomposition arising from a barycentric subdivision. The 
bijection between the cells of $K$ and $K^{*}$ induces an isomorphism $D$ between the homology groups $H_{p}(K)$ and the cohomology groups $H^{k-p}\left(K^{*}\right)$, that is, an isomorphism between $H_{p}(M)$ and $H^{k-p}(M)$. This is just the well-known Poincaré duality.

The intersection of cells is denoted by $I($,$) . It gives rise to a bilinear$ pairing

$$
\phi: H_{k-p}(M) \times H_{k-q}(M) \rightarrow H_{k-p-q}(M),
$$

and we also denote $\phi(a, b)$ by $a \circ b$ for homology classes $a, b$. The cup and cap products are then expressed by

$$
D a \cup D b=D(a \circ b) \text { for } a, b \in H_{*}(M),
$$

and

$$
D a \cap b=a \circ b \text { for } a, b \in H_{*}(M) .
$$

Moreover, if $\langle$,$\rangle denotes the Kronecker pairing between cohomology classes$ and homology classes of the same degree, then

$$
\langle D a, b\rangle=a \circ b \text { for } a \in H_{p}(M), b \in H_{k-p}(M),
$$

and

$$
\langle a \cup \beta, c\rangle=\langle\alpha, \beta \cap c\rangle \text { for } \alpha \in H^{p}(M), \beta \in H^{q}(M), c \in H_{p+q}(M) .
$$

Throughout this paper we will use $\mathbf{R}_{k, l}$ to denote the Grassmannian manifold of oriented $k$-planes through the origin of a $(k+l)$-dimensional Euclidean space $\mathbf{R}^{k+l}$, and $\mathbf{C}_{k, l}$ to denote the Grassmannian manifold of complex $k$-planes through the origin of a $(k+l)$-dimensional complex Euclidean space $\mathbf{C}^{k+l}$. $D$ will denote Poincaré duality in whatever manifold we are dealing with.

A canonical cell decomposition of the Grassmannian manifolds has been studied in full by Wu [17]. Here we summarize the important facts. In the following, all planes are understood to pass through the origin.

To obtain a cell decomposition of $\mathbf{R}_{k, l}$, we fix an orthonormal basis $e_{1}, \cdots, e_{k+l}$ of $\mathbf{R}^{k+l}$, with coordinates $x_{i}(i=1, \cdots, k+l)$, and consider the set of all functions $\omega:\{1, \cdots, k\} \rightarrow \mathbf{Z}$ such that $0 \leq \omega(1) \leq \cdots \leq \omega(k) \leq l$. Such a function is called a Schubert symbol.

Let $\mathbf{R}^{m}$ be the subspace of $\mathbf{R}^{k+l}$ spanned by $e_{1}, \cdots, e_{m}$, for $1 \leq m \leq k+l$. Then the set $U_{\omega}$ of oriented $k$-planes $X$ of $\mathbf{R}^{k+l}$ such that

$$
\operatorname{dim}\left(X \cap \mathbf{R}^{\omega(i)+i}\right) \geq i \quad \text { for } i=1, \ldots, k,
$$

is a pseudomanifold of dimension $d(\omega)=\sum_{i=1}^{k} \omega(i)$. Let $X_{\omega}$ be the $k$-plane 
spanned by $e_{\bar{i}}(i=1, \ldots, k)$, where $\bar{i}=\omega(i)+i$. Let $X_{\omega}^{+}$(respectively $X_{\omega}^{-}$) be the $k$-plane $X_{\omega}$ with the orientation of $\left(e_{I}, \ldots, e_{\bar{k}}\right)$ (respectively the opposite orientation). We now consider the sets $N_{\omega}^{+}\left(\right.$respectively $\left.N_{\omega}^{-}\right)$of oriented $k$-planes of $\mathbf{R}^{k+l}$ having a nondegenerate orthogonal projection on $X_{\omega}$ and whose orientation projects to that of $X_{\omega}^{+}$(respectively $\bar{X}_{\omega}^{-}$). Normal coordinates $a_{i j}$ can be introduced on $N_{\omega}^{+}$and $N_{\omega}^{-}$by the equations

$$
x_{j}=\sum_{i=1}^{k} a_{i j} x_{\bar{i}} \text { for } j=1, \ldots, l
$$

where $\{\tilde{j}\}=\{1, \cdots, \omega(1) ; \omega(1)+2, \cdots, \omega(2)+1 ; \omega(2)+3, \cdots ; \cdots, k+l\}$, and $\{\bar{i}\}=\{\omega(1)+1, \cdots, \omega(k)+k\}$. We orient $N_{\omega}^{+}$and $N_{\omega}^{-}$(which are manifolds diffeomorphic to Euclidean spaces) by the order $\left(a_{11}, \cdots, a_{1 l} ; \cdots ; a_{k 1}, \cdots\right.$, $\left.a_{k l}\right)$. Next we write

$$
U_{\omega}^{+}=N_{\omega}^{+} \cap U_{\omega^{\prime}} \quad U_{\omega}^{-}=N_{\omega}^{-} \cap U_{\omega^{\prime}}
$$

and orient $U_{\omega}^{+}$and $U_{\omega}^{-}$by the order $\left(a_{11}, \cdots, a_{1, \omega(1)}, \cdots, a_{k, \omega(k)}\right)$. In explicit form, the $k$-planes in $U_{\omega}^{+}$and $U_{\omega}^{-}$are spanned by the row vectors of the matrix

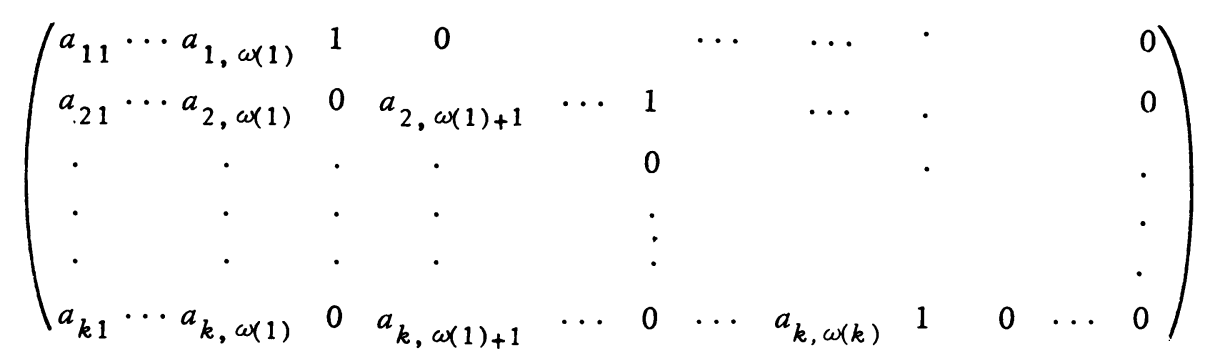

We orient the manifold $\mathbf{R}_{k, l}$ canonically by that of $N_{\omega_{l}}^{+}$, where $\omega_{l}(i)=l$ for $i=1, \cdots, k$. Wu has shown that the orientation of $N_{\omega}^{+}$(respectively $N_{\omega}^{-}$) is coherent or not with $\mathbf{R}_{k, l}$ according as $k(k l-d(\omega))$ (respectively $k(k l-d(\omega))+$ $m+n)$ is even or odd.

We will make one abbreviation in writing $\omega^{ \pm}$instead of $U_{\omega}^{ \pm}$when $\omega$ is given in an explicit form, thus, $\left(b_{1} \cdots b_{k}\right)^{ \pm}$. Also, if any number of zeros occur in the Schubert symbol, we will just omit them and write, for example, (l) instead of $(0 \ldots l)$.

The sets $U_{\omega}^{+}$and $U_{\omega}^{-}$, as $\omega$ runs through all Schubert symbols, form an open cell decomposition of $\mathbf{R}_{k, l}$. Each $U_{\omega}^{+}$and $U_{\omega}^{-}$is accordingly called a Schubert cell. The boundary farmulas are given by [3] 


$$
\begin{aligned}
& \partial\left(b_{1}, \ldots, b_{k}\right)^{ \pm}=\sum(-1)^{b_{1}+\cdots+b_{i}}\left[(-1)^{k-i+1}\left(b_{1}, \ldots, b_{i}-1, \ldots, b_{k}\right)^{ \pm}\right. \\
& \left.+(-1)^{b_{i+1}}\left(b_{1}, \ldots, b_{i}-1, \ldots, b_{k}\right)^{\mp}\right], \\
& \delta\left(b_{1}, \ldots, b_{k}\right)^{ \pm}=\sum(-1)^{b_{1}+\cdots+b_{i}}\left[(-1)^{k-i}\left(b_{1}, \ldots, b_{i}+1, \ldots, b_{k}\right)^{ \pm}\right. \\
& \left.+(-1)^{b_{i+1}}\left(b_{1}, \ldots, b_{i}+1, \ldots, b_{k}\right)^{\mp}\right] \text {. }
\end{aligned}
$$

For any particular $\omega$, let $i_{0}$ be the first jump point and $i_{s}$ the last (that is, $\omega\left(i_{0}\right)<\omega\left(i_{0}+1\right)$, etc. $)$. If $\omega^{+}+(-1)^{k+\omega\left(i_{0}+1\right)+i_{0} \omega^{-}}$is an integral cycle, we denote its homology class by $\left[\omega^{+}+(-1)^{k+\omega\left(i_{0}+1\right)+i 0} \omega^{-}\right]$, and sometimes abbreviate it as $[\omega]$, for example, $[(22)]=\left[(22)^{+}+(22)^{-}\right]$. Likewise, if $\omega^{+}+(-1)^{k+\omega\left(i_{s}\right)+i_{s} \omega^{-}}$is an integral cocycle, we denote its cohomology class by $\left\{\omega^{+}+(-1)^{k+\omega\left(i_{s}\right)+i s} \omega^{-}\right\}$, and sometimes abbreviate it as $\{\omega\}$, for example, $\{(22)\}=\left\{(22)^{+}+(22)^{-}\right\}$.

The Schubert cell decomposition of $\mathbf{R}_{k, l}$ has a dual cell decomposition obtained by defining the Schubert cells relative to the basis $\left(e_{k+l}, \ldots, e_{1}\right)$. Then a Schubert cell $\left(b_{1} \cdots b_{k}\right)$ in the old definition is dual to a Schubert cell $\left(l-b_{k}, \cdots, l-b_{1}\right)$ in the new definition. The appropriate signs can be determined in individual cases.

In the complex case, a cell decomposition can be obtained in a similar way by means of the Schubert symbols, with one significant difference: that there is only one open cell, denoted by $\omega_{0}^{C}$, corresponding to each Schubert symbol $\omega$. This cell is oriented by its complex structure. Each cell is an integral cycle. We will use $\omega^{\mathrm{C}}$ to denote the closure of $\omega_{0}^{\mathrm{C}}$; thus $\omega^{\mathrm{C}}$ is the set of all complex $k$-planes $X$ in $\mathbf{C}^{k+l}$ such that

$$
\operatorname{dim}_{C}\left(X \cap \mathbf{C}^{\omega(i)+i}\right) \geq i \text { for } i=1, \cdots, k .
$$

Homology and cohomology classes are denoted by brackets and braces as in the real case, for example, $\left[(1)^{C}\right],\left\{(1)^{C}\right\}$.

There is a natural oriented $k$-plane bundle over $\mathbf{R}_{k, l}$ which is $(l-1)$ universal, that is, any real oriented $k$-plane bundle over a CW-complex $K$ of dimension $\leq l-1$ is induced by a map $t: K \rightarrow \mathbf{R}_{k, l}$ which is unique up to homotopy. The image under $t^{*}$ of the cohomology ring of $\mathbf{R}_{k, l}$ forms a subring of $H^{*}(K)$ which is independent of $t$ and $l$ (as long as $l>\operatorname{dim} K$ ), and is called the characteristic ring of the bundle. Its elements are called characteristic classes.

Among the cohomology classes of $\mathbf{R}_{k, l}$ are the Euler class

$$
\Omega=\{\underbrace{(1 \ldots 1)}_{k}\},
$$


the Pontrjagin classes

$$
P_{r}=\{\underbrace{(2 \ldots 2)}_{2 r}\}
$$

for $2 r \leq k$, and the dual Pontrjagin classes $\bar{P}_{r}=(-1)^{r}\{(2 r, 2 r)\}$ for $2 r \leq l$. These are important because the Euler class together with the Pontrjagin classes or the dual Pontrjagin classes generate the cohomology ring of $\mathbf{R}_{k, l} \bmod$ torsion up to dimension $l-1$. They are all preserved under the induced homomorphisms of inclusion maps $\mathbf{R}_{k, l} \subset \mathbf{R}_{k, l^{\prime}}$ for $l<l^{\prime}$.

If $K$ is a CW-complex and $t: K \rightarrow \mathbf{R}_{k, l}$ is a map inducing an oriented $k$-plane bundle $\xi$ over $K$, we have the Euler class, the Pontrjagin classes, and the dual Pontrjagin classes of $\xi$ defined respectively by

$$
\begin{array}{ll}
\Omega(\xi)=t^{*} \Omega, & \\
P_{r}(\xi)=t * P_{r} & \text { for } 2 r \leq k, \\
\bar{P}_{r}(\xi)=t * \bar{P}_{r} & \text { for } 2 r \leq l .
\end{array}
$$

If $\xi$ is the tangent bundle of the manifold $M^{(k)}$, it is known that $\Omega(\xi)$ evaluated on the fundamental cycle of $M$ gives the Euler characteristic of $M$. By convention, we also write $\Omega(M)=\Omega(T(M)), P_{r}(M)=P_{r}(T(M)), \bar{P}_{r}(M)=\bar{P}_{r}(T(M))$, where $T(M)$ is the tangent bundle of $M$.

There is also a natural oriented $l$-plane bundle over $\mathbf{R}_{k, l}$, and its Euler class is called the normal Euler class $\tilde{\Omega}$ of $\mathbf{R}_{k, l}$. If $\iota: M^{(k)} \rightarrow \mathbf{R}^{k+l}$ is an immersion with Gauss map $t: M \rightarrow \mathbf{R}_{k, l}$, the normal Euler class $\tilde{\Omega}(\iota)$ of the immersion is defined as $t^{*} \tilde{\Omega}$. It is clearly equal to the Euler class of the normal bundle of $M$ in $\mathbf{R}^{k+l}$. An important fact which will be used very often in this paper is the following: If $\iota$ is an embedding of a compact orientable manifold $M$ in any Euclidean space, then its normal Euler class is zero. A simple proof of this fact can be found in [4].

In the complex case, we define the Chern classes

$$
c_{r}=(-1)^{r}\left\{(\underbrace{1 \ldots 1}_{r})^{c}\right\} \quad(r \leq k)
$$

and the dual Chern classes

$$
\bar{c}_{r}=\left\{(r)^{C}\right\} \quad(r \leq l)
$$

of $\mathrm{C}_{k, i}$. Each of these sets of cohomology classes generates the cohomology ring of $\mathrm{C}_{k, l}$. If $t: K \rightarrow \mathrm{C}_{k, l}$ is a map inducing a complex $k$-plane bundle $\eta$ over $K$, we have the Chern classes and dual Chern classes of $\eta$ defined respectively by 


$$
\begin{aligned}
& c_{r}(\eta)=t^{*} c_{r} \quad \text { for } r \leq k, \\
& \bar{c}_{r}(\eta)=t^{*} \bar{c}_{r} \quad \text { for } r \leq l .
\end{aligned}
$$

Under our normalizations, the highest Chern class $c_{n}(\eta)$ of a complex $n$-plane bundle is equal to the Euler class of the underlying oriented $2 n$-plane bundle $\hat{\eta}$, while the Pontrjagin classes are given by

$$
(-1)^{r} P_{r}(\hat{\eta})=\sum_{j}(-1)^{j} c_{j}(\eta) \cup c_{2 r-j}(\eta) .
$$

The Whitney duality formulas are

$$
\left(\sum_{r \geq 0} P_{r}(\xi)\right) \cup\left(\sum_{s \geq 0} \bar{P}_{s}(\xi)\right)=1 \bmod \text { torsion }
$$

and

$$
\left(\sum_{r \geq 0} c_{r}(\eta)\right) \cup\left(\sum_{s \geq 0} \bar{c}_{s}(\eta)\right)=1 .
$$

4. Homology and cohomology of $\mathbf{R}_{2 m, 2}$. Let $d: \mathbf{R}_{2 m, 2} \rightarrow \mathbf{R}_{2,2 m}$ be the diffeomorphism which sends an oriented $2 m$-plane in $\mathbf{R}^{2 m+2}$ into its oriented orthogonal complement, relative to a fixed Euclidean metric in $\mathbf{R}^{2 m+2}$. We study the induced homomorphism of the cohomology groups.

Propositon 4.1. We bave

$$
d^{*}\left\{(r r)^{+}+(-1)^{r}(r r)^{-}\right\}=(-1)^{r(r-1) / 2}\left\{\left(2_{r}\right)^{+}+(-1)^{r}\left(2_{r}\right)^{-}\right\}
$$

for $1 \leq r \leq 2 m$, where $(2)$ stands for the Schubert symbol $(2 \ldots 2)$ in $\mathbf{R}_{2 m, 2}$ with $r$ twos.

Proof. $\left(2_{r}\right)^{+}$consists of the $2 m$-planes spanned by the row vectors of

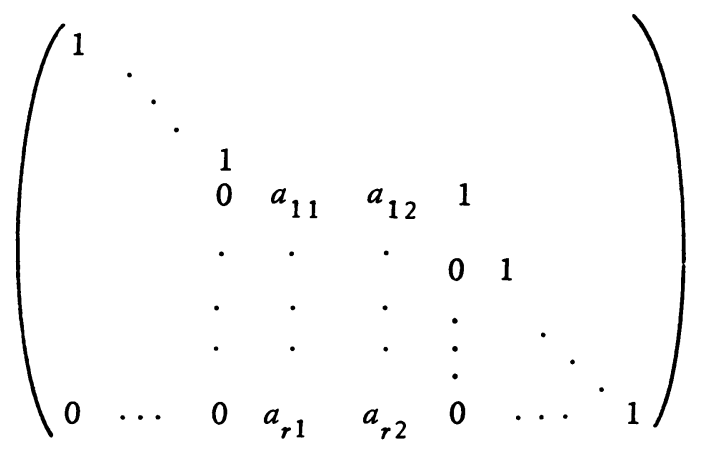

and oriented by the vectors in their natural order. The orthogonal complement of this $2 m$-plane is the 2-plane spanned and oriented by the row vectors of 


$$
\left(\begin{array}{ccccccc}
0 \ldots & \cdots & 0 & -a_{11} & \cdots & -a_{r 1} \\
0 \ldots & 0 & 0 & 1 & -a_{12} & \cdots & -a_{r 2}
\end{array}\right)
$$

Choose coordinates $x_{1}, \ldots, x_{2 m+2}$ and $y_{1}, \ldots, y_{2 m+2}$ for $\mathbf{R}^{2 m+2}$ for the definitions of Schubert varieties in $\mathbf{R}_{2 m, 2}$ and $\mathbf{R}_{2,2 m}$ respectively, such that

$$
\begin{aligned}
y_{j} & =x_{2 m+3-j} \quad \text { for } j=1, \ldots, 2 m+1, \\
y_{2 m+2} & =(-1)^{m+1} x_{1} .
\end{aligned}
$$

Note that $\left(x_{j}\right)$ and $\left(y_{j}\right)$ have the same orientation. $\left(2_{r}\right)^{+}$is oriented by $\left(a_{11}, a_{12}, \ldots, a_{r 1}, a_{r 2}\right)$ and $(r r)-$ by $\left(-a_{r 2}, \ldots,-a_{12},-a_{r 1}, \ldots,-a_{11}\right)$. Thus one sees easily that

$$
d\left(2_{r}\right)^{+}=(-1)^{r(r+1) / 2}(r r)^{-} .
$$

Hence

$$
\begin{aligned}
d^{*}\left\{(r r)^{+}+(-1)^{r}(r r)^{-}\right\} & =(-1)^{r(r+1) / 2}\left\{\left(2_{r}\right)^{-}+(-1)^{r}\left(2_{r}\right)^{+}\right\} \\
& =(-1)^{r(r-1) / 2}\left\{\left(2_{r}\right)^{+}+(-1)^{r}\left(2_{r}\right)^{-}\right\} .
\end{aligned}
$$

Corollary 4.2. The normal Euler class of $\mathbf{R}_{2 m, 2}$ is $\left\{(2)^{+}-(2)^{-}\right\}$.

Proof. Recall that the normal Euler class of $\mathbf{R}_{2 m ; 2}$ is the Euler class of the natural 2-plane bundle over $\mathbf{R}_{2 m_{.2}}$, and is therefore represented by $\left\{(11)^{+}-(11)^{-}\right\}$in $\mathbf{R}_{2,2 m}$.

Lemma 4.3. In $\mathbf{R}_{2 m, 2},\left[\left(2_{2 m-r}\right)\right] \circ\left[\left(2_{2 m-s}\right)\right]=\left[\left(2_{2 m-r-s}\right)\right]$ for $1 \leq r, s$ $\leq 2 m, r+s \leq 2 m$.

Proof. We define $\left(2_{2 m-r-s}\right),\left(2_{2 m-r}\right),\left(2_{2 m-s}\right)$ in terms of coordinate systems $\left(x_{j}\right),\left(x_{j}^{\prime}\right),\left(x_{j}^{\prime \prime}\right)$ respectively, related by

$$
\begin{gathered}
x_{j}^{\prime}= \begin{cases}x_{j} \quad \text { for } j=1, \ldots, r \text { or } r+s+3, \cdots, 2 m+2, \\
x_{j+s} \text { for } j=r+1, r+2, \\
x_{j-2} \text { for } j=r+3, \ldots, r+s+2,\end{cases} \\
x_{j}^{\prime \prime}= \begin{cases}x_{j+r} & \text { for } j=1, \cdots, s+2, \\
x_{j-s-2} & \text { for } j=s+3, \cdots, r+s+2, \\
x_{j} & \text { for } j=r+s+3, \cdots, 2 m+1, \\
(-1)^{r s} x_{2 m+2} & \text { for } j=2 m+2 .\end{cases}
\end{gathered}
$$

These coordinate systems all have the same orientation. Let $\left(e_{j}\right)$ be the basis 
of $\mathbf{R}^{2 m+2}$ corresponding to $\left(x_{j}\right)$. The central element of $\left(2_{2 m-r}\right)^{+}$is spanned and oriented by $\left\{e_{1}, \cdots, e_{r}, e_{r+1}, \cdots, e_{r+s}, e_{r+s+3}, \cdots, e_{2 m+2}\right\}$. The central element of $\left(2_{2 m-s}\right)^{+}$is spanned and oriented by $\left\{e_{r+1}, \ldots, e_{r+s}, e_{1}, \ldots, e_{r}\right.$, $\left.e_{r+s+3}, \cdots, e_{2 m+1},(-1)^{r s} e_{2 m+2}\right\}$, which is the same as $\left\{e_{1}, \cdots, e_{r+s}\right.$, $\left.e_{r+s+3}, \cdots, e_{2 m+2}\right\}$. Therefore the geometric intersection of $\left(2_{2 m-r}\right)^{+}$and $\left(2_{2 m-s}\right)^{+}$is $\left(2_{2 m-r-s}\right)^{+}$. The sign of the intersection is easily verified to be +1 . Similarly for $\left(2_{2 m-r}\right)^{-}$and $\left(2_{2 m-s}\right)^{-}$. Therefore

$$
\begin{array}{r}
{\left[\left(2_{2 m-r}\right)^{+}+(-1)^{2 m-r}\left(2_{2 m-r}\right)^{-}\right] \circ\left[\left(2_{2 m-s}\right)^{+}+(-1)^{2 m-s}\left(2_{2 m-s}\right)^{-}\right]} \\
=\left[\left(2_{2 m-r-s}\right)^{++}+(-1)^{2 m-r-s}\left(2_{2 m-r-s}\right)^{-}\right] .
\end{array}
$$

Proposition 4.4. In $H^{*}\left(\mathbf{R}_{2 m, 2}\right),\{(2)\}^{r}=\{(2)\}$ for $1 \leq r \leq 2 m$.

Proof. From Lemma 4.3, it follows in particular that $\{(2)\}$ is dual to $\left[\left(2_{2 m-r}\right)\right]$. Hence

$$
D\left(\left\{\left(2_{r}\right)\right\} \cup\left\{\left(2_{s}\right)\right\}\right)=\left[\left(2_{2 m-r}\right)\right] \circ\left[\left(2_{2 m-s}\right)\right]=\left[\left(2_{2 m-r-s}\right)\right] .
$$

This means that $\left\{\left(2_{r}\right)\right\} \cup\left\{\left(2_{s}\right)\right\}=\left\{\left(2_{r+s}\right)\right\}$ for $r+s \leq 2 m$. By induction it follows that $\{(2)\}^{r}=\left\{\left(2_{r}\right)\right\}$.

Let $\Omega, \widetilde{\Omega}$ be respectively the Euler class and normal Euler class of $\mathbf{R}_{2 m, 2^{*}}$ It is known that the cohomology ring of $\mathbf{R}_{2 m, 2}$ is generated by $\tilde{\Omega}$ and a class $v \in H^{2 m}$ such that $\Omega+\widetilde{\Omega}^{m}=2 v$, and that a basis of the cohomology groups is given by $\left\{\widetilde{\Omega}^{r}, v \cup \widetilde{\Omega}^{r}\right\}, r=0,1, \cdots, m$ (for example, see [7]). We are now going to identify these generators in terms of Schubert cocycles.

For $0 \leq r \leq m$, let $a_{r}$ be the Schubert symbol $(1 \cdots 12 \cdots 2)$ with $(2 m-2 r)$ ones and $r$ twos, of dimension $2 m$.

Proposition 4.5. The bomology classes $z_{1}=\left[a_{m}^{+}+(-1)^{m} a_{m}^{-}\right]$and $z_{2}=$ $\left[\Sigma_{r=0}^{m}(-1)^{r} a_{r}^{+}\right]$form a basis of $H_{2 m}\left(\mathbf{R}_{2 m, 2}\right)$, and the cobomology classes $\zeta_{1}=\left\{a_{m}^{+}+(-1)^{m} a_{m}^{-}\right\}$and $\zeta_{2}=\left\{\sum_{r=0}^{m} a_{r}^{+}\right\}$form a basis of $H^{2 m}\left(\mathbf{R}_{2 m, 2}\right)$.

Proof. Let $b_{r}$ (respectively $c_{r}$ ) be the Schubert symbol $(1 \cdots 12 \cdots 2)$ with $2 m-2 r-1$ (respectively $2 m-2 r+1$ ) ones and $r$ twos, for $0 \leq r \leq m-1$ (respectively $1 \leq r \leq m$ ). Their dimensions are $2 m-1$ and $2 m+1$ respectively. We also put $b_{-1}=b_{m}=c_{0}=c_{m+1}=0$. Then, using formula (3.1), we get

$$
\begin{aligned}
& \partial a_{r}^{+}=(-1)^{r+1} b_{r}^{+}-b_{r}^{-}+(-1)^{r} b_{r-1}^{+}-b_{r-1}^{-}, \text {and } \\
& \partial a_{r}^{-}=-b_{r}^{+}+(-1)^{r+1} b_{r}^{-}-b_{r-1}^{+}+(-1)^{r} b_{r-1}^{-}, \quad 0 \leq r \leq m .
\end{aligned}
$$


Therefore

$$
\partial\left(a_{m}^{+}+(-1)^{m} a_{m}^{-}\right)=0, \quad \partial\left(\sum_{r=0}^{m}(-1)^{r} a_{r}^{+}\right)=0 .
$$

Consequently $a_{m}^{+}+(-1)^{m} a_{m}^{-}$and $\sum_{r=0}^{m}(-1)^{r} a_{r}^{+}$are cycles. Likewise

$$
\begin{aligned}
& \delta a_{r}^{+}=(-1)^{r} c_{r}^{+}-c_{r}^{-}+(-1)^{r} c_{r+1}^{+}+c_{r+1}^{-}, \text {and } \\
& \delta a_{r}^{-}=-c_{r}^{+}+(-1)^{r} c_{r}^{-}+c_{r+1}^{+}+(-1)^{r} c_{r+1}^{-}, \quad 0 \leq r \leq m,
\end{aligned}
$$

and we conclude that $a_{m}^{+}+(-1)^{m} a_{m}^{-}$and $\sum_{r=0}^{m} a_{m}^{+}$are cocycles. To show that $z_{1}, z_{2}$ form a basis of $H_{2 m}\left(\mathbf{R}_{2 m, 2}\right)$ and $\zeta_{1}, \zeta_{2}$ a basis of $H^{2 m}\left(\mathbf{R}_{2 m, 2}\right)$, we only need to evaluate the determinant of their Kronecker products.

$$
\begin{aligned}
\left|\left\langle\zeta_{1}, z_{1}\right\rangle\left\langle\zeta_{2}, z_{1}\right\rangle\right| & =\left|\begin{array}{cc}
2 & 1 \\
\left\langle\zeta_{1}, z_{2}\right\rangle\left\langle\zeta_{2}, z_{2}\right\rangle
\end{array}\right| \\
& =\left\{\begin{array}{cc}
\mid-1)^{m} & \sum_{r=0}^{m}(-1)^{r} \\
1 & 1
\end{array} \mid\right. \\
\left|\begin{array}{cc}
2 & 1 \\
-1 & 0
\end{array}\right| & m \text { odd, } \\
& =1 .
\end{aligned}
$$

Then since $H_{2 m}\left(\mathbf{R}_{2 m, 2}\right)$ and $H^{2 m}\left(\mathbf{R}_{2 m, 2}\right)$ are both isomorphic to $\mathbf{Z} \oplus \mathbf{Z}$ and are dual under the Kronecker pairing, it follows that the elements in question actually form bases.

Proposition 4.6. $\left\{a_{0}^{+}-a_{0}^{-}\right\}=-\zeta_{1}+2 \zeta_{2}$.

Proof. One could prove by evaluating both sides on the basis of $H_{2 m}\left(\mathbf{R}_{2 m, 2}\right)$ but here we give a direct verification. With $b_{r}$ as in Proposition 4.1,

$$
\delta b_{r}^{+}=(-1)^{r+1} a_{r}^{+}-a_{r}^{-}+(-1)^{r+1} a_{r+1}^{+}-a_{r+1}^{-}, \quad 0 \leq r \leq m-1 .
$$

Therefore,

$$
\begin{aligned}
\sum_{r=0}^{m-1}(-1)^{r} \delta b_{r}^{+} & =-\sum_{r=0}^{m-1} a_{r}^{+}-\sum_{r=1}^{m} a_{r}^{+}-a_{0}^{-}+(-1)^{m} a_{m}^{-} \\
& =-2 \sum_{r=0}^{m} a_{r}^{+}+a_{0}^{+}+a_{m}^{+}-a_{0}^{-}+(-1)^{m} a_{m}^{-}
\end{aligned}
$$


and so

$$
\left\{a_{0}^{+}-a_{0}^{-}\right\}=-\left\{a_{m}^{+}+(-1)^{m} a_{m}^{-}\right\}+2\left\{\sum_{r=0}^{m} a_{r}^{+}\right\}
$$

Note. From this proposition it follows that $\zeta_{2}$ is the class $v$ mentioned above.

Suppose a complex structure in $\mathbf{R}^{2 m+2}$ is given. The complex projective space $\mathbf{C}_{m, 1}$ is then naturally embedded in $\mathbf{R}_{2 m, 2}$ as the set of all complex $m$-planes in $\mathbf{C}^{m+1}=\mathbf{R}^{2 m+2}$. Let $\kappa$ be the $2 m$-dimensional cohomology class in $\mathbf{R}_{2 m, 2}$ dual (Poincaré) to the homology class represented by the embedded $\mathbf{C}_{m, 1}$ in $\mathbf{R}_{2 m, 2}$.

Lemma 4.7. $\kappa=\left\{\sum_{r=0}^{m} a_{r}^{+}\right\}=\zeta_{2}$.

Proof. By Proposition 4.5, one only needs to evaluate $\kappa, \zeta_{2}$ on the basis $\left\{z_{1}, z_{2}\right\}$ of $H_{2 m}\left(\mathbf{R}_{2 m, 2}\right)$ and verify that the values are equal. Thus we wish to find the intersections of $\mathbf{C}_{m, 1}$ with the Schubert cells of $\mathbf{R}_{2 m, 2}$. Choose a coordinate system $\left\{z_{1}, \cdots, z_{m+1}\right\}$ in $\mathbf{C}^{m+1}$ with basis vectors $\left\{f_{1}, \cdots, f_{m+1}\right\}$, and a coordinate system $\left\{x_{1}, \cdots, x_{2 m+2}\right\}$ of the underlying $\mathbf{R}^{2 m+2}$, with basis vectors $\left\{e_{1}, \cdots, e_{2 m+2}\right\}$, related by

$$
z_{j}=x_{j}+\sqrt{-1} x_{2 m-j+3} \text { for } j=1, \ldots, m+1 .
$$

The orientations are clearly the same.

Let $A$ be the $2 m$-cycle in $\mathbf{R}_{2 m, 2}$ which is the embedded $\mathbf{C}_{m, 1}$. It is clear that $A \cap a_{r}^{-}=\emptyset$ for $r=0, \cdots, m$. For a given $r=0, \cdots, m$, suppose an oriented $2 m$-plane $X_{r}$ belongs to $A \cap a_{r}^{+}$. Recall that

$$
a_{r}=(\underbrace{1 \cdots 1}_{2 m-2 r} \underbrace{2 \ldots 2}_{r}),
$$

so from the definition of the Schubert cell,

$$
\mathbf{R}^{r} \subset X_{r}
$$

and

$$
\operatorname{dim}\left(X_{r} \cap \mathbf{R}^{2 m-r+1}\right) \geq 2 m-r,
$$

where $\mathbf{R}^{j}$ is spanned by $e_{1}, \cdots, e_{j}$. Since $X_{r} \in A$, it follows from (4.2) that $X_{r}$ contains $e_{1}, \cdots, e_{r}$ and $e_{2 m+3-r}, \cdots, e_{2 m+2}$. Let $\mathbf{R}_{*}^{j}$ be spanned by $e_{2 m+3-j}, \cdots, e_{2 m+2}$. Then it follows from (4.3) by taking the complex conjugate that

$$
\operatorname{dim}\left(X_{r} \cap \mathbf{R}_{*}^{2 m-r+1}\right) \geq 2 m-r
$$


Now the dimension theorem for vector spaces yields

$$
\begin{aligned}
\operatorname{dim}\left(X_{r} \cap \mathbf{R}^{2 m-r+1} \cap \mathbf{R}_{*}^{2 m-r+1}\right)+\operatorname{dim}\left(X_{r} \cap \mathbf{R}^{2 m-r+1}+X_{r} \cap \mathbf{R}_{*}^{2 m-r+1}\right) \\
=\operatorname{dim}\left(X_{r} \cap \mathbf{R}^{2 m-r+1}\right)+\operatorname{dim}\left(X_{r} \cap \mathbf{R}_{*}^{2 m-r+1}\right) .
\end{aligned}
$$

Note that $\mathbf{R}^{2 m-r+1}+\mathbf{R}_{*}^{2 m-r+1}=\mathbf{R}^{2 m+2}$ for $r \leq m$. Therefore (4.3), (4.4) and (4.5) together give

$$
\operatorname{dim}\left(X_{r} \cap \mathbf{R}^{2 m-r+1} \cap \mathbf{R}_{*}^{2 m-r+1}\right) \geq(2 m-r)+(2 m-r)-2 m=2 m-2 r .
$$

The subspace $\mathbf{R}^{2 m-r+1} \cap \mathbf{R}_{*}^{2 m-r+1}$ is spanned by $e_{r+2}, \cdots, e_{2 m-r+1}$ and is of dimension $2 m-2 r$. Thus we have shown that $X_{r}$ also contains $e_{r+2}, \cdots$, $e_{2 m-r+1}$, so $X_{r}$ is the central element of $a_{r}^{+}$. Therefore $A \cap a_{r_{r}}^{+}$consists of only one element, the central element of $a_{r}^{+}$. We now find the sign of the intersection.

Let $N_{r}^{+}$be the neighborhood of $X_{r}$ in $\mathbf{R}_{2 m, 2}$ defined by the coordinates $a_{i j}, i=1, \cdots, 2 m$ and $j=1,2$, such that

$$
x \underset{j}{\sim}=\sum_{i=1}^{2 m} a_{i j} x_{\vec{i}}
$$

where $\{\bar{i}\}=\{1, \cdots, r, r+2, \cdots, 2 m-r+1,2 m-r+3, \cdots, 2 m+2\}$, and $\{\tilde{j}\}=\{r+1,2 m-r+2\}$. Let $N_{r}^{+}$be oriented by $\left\{a_{11}, a_{12}, a_{21} ; a_{22}, \cdots\right.$, $\left.a_{2 m, 1}, a_{2 m, 2}\right\}$. It has been shown by Wu [17] that this orientation is the same as that in $\mathbf{R}_{2 m, 2}$. In a neighborhood of $X_{r}$, the $2 m$-planes which are elements of $A$ are defined by $z_{r+1}=\sum_{i=1}^{m} b_{i} z_{i}$. Write $b_{i}=b_{i}^{\prime}+\sqrt{-1} b_{i}^{\prime \prime}$. Then

$$
x_{r+1}+\sqrt{-1} x_{2 m-r+2}=\sum_{i=1}^{m}\left(b_{i}^{\prime}+\sqrt{-1} b_{i}^{\prime \prime}\right)\left(x_{i}+\sqrt{-1} x_{2 m-\bar{i}+3}\right) .
$$

Therefore,

$$
\begin{aligned}
& x_{\tilde{1}}^{\tilde{\tau}}=x_{r+1}=\sum_{i+1}^{m}\left(b_{i}^{\prime} x_{\bar{i}}-b_{i}^{\prime \prime} x_{2 m-\bar{i}+3}\right), \\
& x_{\tilde{2}}=x_{2 m-r+2}=\sum_{i=1}^{m}\left(b_{i}^{\prime \prime} x_{\bar{i}}+b_{i}^{\prime} x_{2 m-\bar{i}+3}\right) .
\end{aligned}
$$

Let $v_{i j}$ be the basis vectors (in $\mathbf{R}^{4 m}$ ) corresponding to the coordinates $a_{i j}$ introduced in $N_{r}^{+}$. Then $A$ is oriented by the vectors

$$
\left\{v_{1,1}+v_{2 m, 2}, v_{1,2}-v_{2 m, 1} ; v_{2,1}+v_{2 m-1,2}, v_{2,2}-v_{2 m-1,1} ;\right.
$$

corresponding to the order $\left\{b_{1}^{\prime}, b_{1}^{\prime \prime} ; b_{2}^{\prime}, b_{2}^{\prime \prime} ; \cdots ; b_{m}^{\prime}, b_{m}^{\prime \prime}\right\}$. On the other hand, $a_{r}^{+}$is oriented by the vectors 


$$
\begin{array}{r}
\left\{v_{r+1,1}, v_{r+2,1}, \cdots, v_{2 m-r, 1} ; v_{2 m-r+1,1}, v_{2 m-r+1,2} ;\right. \\
\left.\ldots ; v_{2 m, 1}, v_{2 m, 2}\right\} .
\end{array}
$$

From (4.6) and (4.7) it follows by straightforward calculations that the index of intersection $I\left(A, a_{r}^{+}\right)=1$. Consequently,

$$
\begin{gathered}
\left\langle\kappa,\left[a_{m}^{+}+(-1)^{m} a_{m}^{-}\right]\right\rangle=I\left(A, a_{m}^{+}\right)+(-1)^{m} I\left(A, a_{m}^{-}\right)=1=\left\langle\zeta_{2},\left[a_{m}^{+}+(-1)^{m} a_{m}^{-}\right]\right\rangle, \\
\left\langle\kappa,\left[\sum_{r=0}^{m}(-1)^{r} a_{r}^{+}\right]\right\rangle=\sum_{r=0}^{m}(-1)^{r} I\left(A, a_{r}^{+}\right)=\sum_{r=0}^{m}(-1)^{r}=\left\langle\zeta_{2},\left[\sum_{r=0}^{m}(-1)^{r} a_{r}^{+}\right]\right\rangle .
\end{gathered}
$$

Thus $\kappa$ and $\zeta_{2}$ have the same values on a homology basis of $\mathbf{R}_{2 m, 2}$ (Proposition 4.5), and hence they are equal.

Theorem 4.8. Let $\Omega, \widetilde{\Omega}$ be the Euler class and normal Euler class of $\mathbf{R}_{2 m, 2}$, and let $\kappa$ be the Poincare dual of the homology class represented by a natural embedding of $\mathbf{C}_{m, 1}$ in $\mathbf{R}_{2 m, 2^{*}}$ Then $\left\{\tilde{\Omega}^{r}, \kappa \cup \widetilde{\Omega}^{r}\right\}, r=0, \cdots, m$, forms a basis of $H^{*}\left(\mathbf{R}_{2 m, 2}\right)$, and $\Omega+\widetilde{\Omega}^{m}=2 \kappa$.

Proof. By Corollary 4.2 and Proposition 4.4, $\widetilde{\Omega}^{m}=\left\{\left(2_{m}\right)\right\}=\zeta_{1}$. The theorem now follows from Lemma 4.7 and Proposition 4.6.

\section{Applications.}

I. Index of RC-singularity. Let $\iota$ be an immersion of a $(2 n-2)$-dimensional compact, orientable manifold $M$ in $\mathbf{C}^{n}$. There is then induced a Gauss map $t: M \rightarrow \mathbf{R}_{2 m, 2}$, where $m=n-1$. The pullbacks of the natural $2 m$-plane and 2-plane bundles over $\mathbf{R}_{2 m, 2}$ by $t$ are precisely the tangent and normal bundles of $M$ in $\mathrm{C}^{n}$ respectively, and so $t^{*} \Omega$ and $t^{*} \tilde{\Omega}$ are the Euler class and normal Euler class of $M$ in $\mathrm{C}^{n}$. If $\iota$ is an embedding, then it is known that $t^{*} \widetilde{\Omega}=0$ (see, for example, [4]). Therefore, from Theorem 4.8, $t^{*} \Omega=2 t^{*} \kappa$.

In particular, this implies a theorem of Seifert [9]: If a compact orientable manifold of dimension $2 m$ can be embedded in a Euclidean space $\mathbf{R}^{2 m+2}$, then its Euler characteristic is even. Moreover, here we have an interpretation of this even number, as follows:

Recall that a point $x \in M$ is called an RC-singular point of $\iota$ if

$$
\operatorname{dim}_{\mathrm{C}} \iota_{*} T_{x}(M) \cap J \iota_{*} T_{x}(M)>n-2 .
$$

In this case, this is equivalent to $\iota_{*} T_{x}(M)$ being a complex subspace of $\mathrm{C}^{n}$, or to $t(x) \in \mathbf{C}_{m, 1} \cup \mathbf{C}_{m, 1}^{-}$, where $\mathbf{C}_{m, 1}^{-}$consists of the negatively oriented complex $m$-dimensional subspaces of $\mathbf{C}^{m+1}$. Let $\mathbf{C}_{m, 1}^{-}$have the opposite orientation to that induced from $\mathrm{C}_{m, 1}$ by the correspondence $\mathrm{C}_{m, 1} \leftrightarrow \mathrm{C}_{m, 1}^{-}$which sends each plane into the one with the opposite orientation. 
Definition 4.9. The index of RC-singularity at $x \in M$ is the index of local intersection of $t(M)$ with $\mathbf{C}_{m, 1} \cup \mathbf{C}_{m, 1}^{-}$in $\mathbf{R}_{2 m, 2}$.

Theorem 4.10. For an embedding 1 of a compact orientable manifold $M^{(2 n-2)}$ in $\mathbf{C}^{n}$, the sum of the indices of RC-singularity over the set of RC-singular points where the tangent spaces bave the complex orientation is equal to the corresponding sum for the opposite orientation, and eacb is equal to balf the Euler characteristic of $M$.

Proof. From Theorem 4.8, $t^{*} \Omega=2 t^{*} \kappa$. Evaluated on $M, t^{*} \Omega$ gives the Euler characteristic, and

$$
\left\langle t^{*} \kappa,[M]\right\rangle=\left\langle\kappa, t_{*}[M]\right\rangle
$$

which is the sum of indices over the set of RC-singular points where the tangent spaces have the complex orientation. For the other sum, let $\nu: \mathbf{R}_{2 m, 2} \rightarrow \mathbf{R}_{2 m, 2}$ be the map which reverses orientations of $2 m$-planes. Then

$$
\begin{aligned}
t^{*} \Omega & =-(\nu \circ t)^{*} \Omega=-2(\nu \circ t)^{*} \kappa, \\
-\left\langle(\nu \circ t)^{*} \kappa,[M]\right\rangle & =-\left\langle\nu^{*} \kappa, t_{*}[M]\right\rangle=\phi\left(\mathbf{C}_{m, 1}^{-}, t_{*}[M]\right) .
\end{aligned}
$$

II. A result of Chern and Spanier. In the case $m=1$, a result essentially the same as Theorem 4.8 has been deduced by Bishop [1] from a theorem of Chern and Spanier [4], but in a different approach. It may be worthwhile to know the relation between the two methods.

Following Chern and Spanier [4], we choose an oriented set of orthonormal basis vectors $e_{1}, \cdots, e_{4}$ in $\mathbf{R}^{4}$. For any oriented 2-plane $X \subset \mathbf{R}^{4}$, choose an oriented orthonormal basis $\left\{f_{1}, f_{2}\right\}$ of $X$. Write

$$
\begin{aligned}
& f_{1} \wedge f_{2}=\sum_{i<j} a_{i j} e_{i} \wedge e_{j}, \\
& x_{1}=a_{12}+a_{34}, \quad x_{2}=a_{23}+a_{14}, \quad x_{3}=-a_{13}+a_{24} \text {, } \\
& y_{1}=a_{12}-a_{34}, \quad y_{2}=a_{23}-a_{14}, \quad y_{3}=-a_{13}-a_{24} \text {. }
\end{aligned}
$$

Then it is shown that $\mathbf{R}_{2,2}$ is diffeomorphic to $S_{1} \times S_{2}$, where $S_{1}, S_{2}$ are the unit spheres in the $x$-space and $y$-space respectively. Orient $S_{1}, S_{2}$ by the induced orientations from $\left(x_{1}, x_{2}, x_{3}\right)$ and $\left(y_{1}, y_{2}, y_{3}\right)$ respectively. Then it is easily checked that our orientation of $\mathbf{R}_{2,2}$ is the same as the product orientation of $S_{1} \times S_{2}$. Let $A$ be the embedded image of $\mathbf{C}_{1,1}$ in $\mathbf{R}_{2,2}$ relative to a complex structure on $\mathbf{R}^{4}$ with the same orientation. Bishop showed that $A=(1,0,0) \times$ $S_{2}$. The orientations are, however, opposite. Thus, if we abbreviate $(1,0,0) \times$ $S_{2}$ into $S_{2}$ and $S_{1} \times(1,0,0)$ into $S_{1}$, we have, from Lemma 4.7, 


$$
S_{2}=-A=-D \zeta_{2},
$$

where $D$ is the Poincare duality in $\mathbf{R}_{2,2}$. It can be shown that, in the notations of Proposition 4.5,

$$
D \zeta_{1}=z_{1}, \quad D \zeta_{2}=-z_{2},
$$

and that $z_{1} \circ z_{1}=2, z_{1} \circ z_{2}=-1, z_{2} \circ z_{2}=0$. From these and the conditions $S_{1} \circ S_{2}=1, S_{1} \circ S_{1}=0$ in $S_{1} \times S_{2}$, it follows that

$$
S_{1}=-z_{1}-z_{2}, \quad S_{2}=z_{2} \text {. }
$$

Consequently, $S_{1}^{*}=D S_{1}=-\zeta_{1}+\zeta_{2}, S_{2}^{*}=D S_{2}=-\zeta_{2}, s_{1}^{*}+S_{2}^{*}=-\zeta_{1}=-\tilde{\Omega}$, $s_{1}^{*}-S_{2}^{*}=-\zeta_{1}+2 \zeta_{2}=\Omega$ (using Proposition 4.6). Thus the theorem of Chern and Spanier becomes

$$
t_{*}(M)=1 / 2 \chi_{M}\left(-S_{1}+S_{2}\right) .
$$

The intersection $t_{*}(M) \circ A=1 / 2 \chi_{M}\left(-S_{1}+S_{2}\right) \circ\left(-S_{2}\right)=1 / 2 \chi_{M}$, as in Theorem 4.9. III. Gaussian curvature of bypersurfaces in $\mathbf{R}^{2 m+1}$.

Lemma 4.11. Let $\iota$ be an immersion of $M^{(2 m)}$ in $\mathbf{R}^{2 m+1} \subset \mathbf{R}^{2 m+2}$, and let a complex structure and a complex Euclidean metric be introduced in $\mathbf{R}^{2 m+2}$. Then at any RC-singular point of the immersion, the index of RC-singularity is equal to the sign of the Gaussian curvature (that is, the product of the principal curvatures) of the bypersurface at the same point, provided that the latter is nonzero.

Proof. Let $\left\{e_{1}, \ldots, e_{2 m+2}\right\}$ be an oriented orthonormal basis in $\mathbf{R}^{2 m+2}$, and $\left\{x_{1}, \cdots, x_{2 m+2}\right\}$ the corresponding coordinate system. Let $\mathbf{R}^{2 m+1}$ be spanned by the first $2 m+1$ basis vectors. Let the complex structure in $\mathbf{R}^{2 m+2}$ be defined by $e_{2 j}=\sqrt{-1} e_{2 j-1}$ for $j=1, \cdots, m+1$. In a neighborhood of an RC-singular point $p \in M$ at which the tangent plane is spanned and oriented by $\left(e_{1}, \cdots, e_{2 m}\right)$, the hypersurface can be expressed in the form $x_{2 m+1}=$ $f\left(x_{1}, \cdots, x_{2 m}\right)$, and it is well known that the Gaussian curvature at $p$ is $K(p)=\operatorname{det}\left(f_{i j}(p)\right)$, where the subscripts denote partial derivatives with respect to $x_{i}$, and $i, j=1, \cdots, 2 m$.

Choose the oriented basis $\left(e_{\cdot 2 m+1} ; e_{1}, \cdots, e_{2 m} ; e_{2 m+2}\right)$ for defining the Schubert varieties in $\mathbf{R}_{2 m, 2}$. A neighborhood $N^{+}$of $(1 \cdots 1)^{+}$(2m factors) in $\mathbf{R}_{2 m, 2}$ is defined by coordinates $a_{i j}, i=1, \cdots, 2 m$ and $j=1,2$, as in the proof of Lemma 4.7. Let $v_{i j}$ be basis vectors corresponding to the coordinates $a_{i j}$ of $\mathbf{R}^{4 m}$. Then $t(M)$ in a neighborhood of $t(p)$ is spanned and oriented by $\left(\sum_{i=1}^{2 m} f_{i 1} v_{i 1}, \cdots, \Sigma_{i=1}^{2 m} f_{i, 2 m} v_{i 1}\right)$. From this it follows that, at the point $p$, 


$$
\begin{gathered}
I\left(t_{*} M, \mathbf{C}_{m, 1}\right)=\operatorname{sgn} \operatorname{det}\left(\sum_{i=1}^{2 m} f_{i 1} v_{i 1}, \ldots, \sum_{i=1}^{2 m} f_{i, 2 m} v_{i 1},\right. \\
v_{11}+v_{22}, v_{12}-v_{21}, \cdots, \\
\left.v_{2 m-1,1}+v_{2 m, 2}, v_{2 m-1,2}-v_{2 m, 1}\right)
\end{gathered}
$$

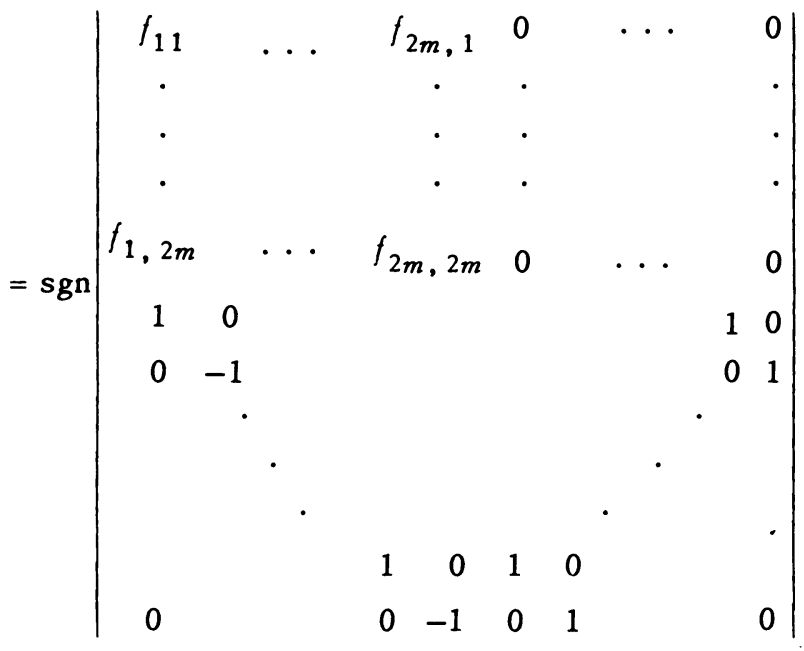

$$
\begin{aligned}
& =\operatorname{sgn} \operatorname{det}\left(f_{i j}\right)=\operatorname{sgn} K(p) \text {. }
\end{aligned}
$$

Similar arguments hold for an RC-singular point where the tangent plane is oppositely oriented.

Theorem 4.12. Let $\iota$ be an embedding of a compact orientable 2m-dimensional manifold in $\mathbf{R}^{2 m+1}$. Let $\chi$ be the Euler characteristic of $M$. Then for any $2 m$-plane $X$ in $\mathbf{R}^{2 m+1}$ there are at least $|X|$ points on $M$ where the tangent plane is parallel to $X$ and where the Gaussian curvature is $\geq 0$ or $\leq 0$ according as $\chi \geq 0$ or $\leq 0$.

Proof. Given $X$, we can choose an orthonormal basis $\left\{e_{1}, \cdots, e_{2 m+1}\right\}$ of $\mathbf{R}^{2 m+1}$ such that $X$ is parallel to the coordinate plane spanned by $e_{1}, \cdots, e_{2 m}$. Extend $\mathbf{R}^{2 m+1}$ to $\mathbf{R}^{2 m+2}$ by adding a vector $e_{2 m+2}$, and introduce a complex structure in $\mathbf{R}^{2 m+2}$ by $e_{2 j}=\sqrt{-1} e_{2 j-1}$ for $j=1, \cdots, m+1$. Then by Theorem 4.10, there are at least $|\chi| \mathbf{R C}$-singular points at which the index of RC-singularity has the same sign as $\chi$. At such a point the Gaussian curvature is either zero or has the same sign as $\chi$, by Lemma 4.11. The theorem therefore follows.

Remark. In the case where the Gaussian curvature is nowhere zero, Theorem 4.12 also follows from Morse theory, because then the critical points of the height function (corresponding to the tangent plane being parallel to $X$ ) are non- 
degenerate, and the parity of the index is determined by the sign of the Gaussian curvature. Then since the sum of the indices is the Euler characteristic of $M$, the theorem follows. Our proof here takes care of the degenerate critical points.

IV. A note on bulls of bolomorpby. Using the observation of Bishop that a generic embedding of the 2-sphere in $\mathrm{C}^{2}$ has at least two RC-singular points where the Hessian is nondegenerate of "elliptic type" (which also follows from Theorem 4.10), Hunt [6] showed that there is a dense set of embeddings of the 2-sphere in $\mathrm{C}^{2}$ such that the hull of holomorphy of the embedded sphere contains a 3-dimensional manifold. Using Theorem 4.10 and an extension theorem of Hunt, similar results might be proved for embeddings of compact orientable manifolds $M^{(2 m)}$ with positive Euler characteristic in $\mathbf{R}^{2 m+2}$, but such investigations are rendered pointless by the following theorem of Wells [16]: Any compact submanifold of real codimension 2 in a Stein manifold of dimension $>2$ has envelope of holomorphy containing a submanifold of one higher dimension.

5. The characteristic formula for $\xi^{(2 n-2)} \oplus \xi^{(2)}=\eta_{(n)}$. Let $\xi \oplus \xi^{\prime}=\eta$ be a decomposition of a complex $n$-plane bundle $\eta$ over $M$ into an oriented $(2 n-2)$-plane bundle $\xi$ and an oriented 2-plane bundle $\xi^{\prime}$. Let $G_{k, 2}(\eta)$ be the total space of the bundle with fibre $\mathbf{R}_{k, 2}$ associated to $\eta$, where $k=2 n-2$. Let $K_{\eta}$ be the subset of $G_{k, 2}(\eta)$ consisting of the complex $(n-1)$-planes of the fibres of $\eta$. If $\eta$ is the universal bundle over the complex Grassmannian $\mathrm{C}_{n, q}$ where $q$ is large, we also write $G_{k, 2}\left(\mathrm{C}_{n, q}\right)$ for $G_{k, 2}(\eta)$ and $K_{0}$ for $K_{\eta}$. We now study the fibre bundle

$$
\pi: G_{k, 2}\left(\mathbf{C}_{n, q}\right) \rightarrow \mathbf{C}_{n, q}
$$

Proposition 5.1. There exists a homomorphism of graded groups $\theta: H^{*}\left(\mathbf{R}_{k, 2}\right)$ $\rightarrow H^{*}\left(G_{k, 2}\left(\mathbf{C}_{n, q}\right)\right)$ such that $\theta\left(\widetilde{\Omega}^{r}\right)=\theta(\widetilde{\Omega})^{r}$ for $0 \leq r \leq n-1, \theta(\kappa)=D K_{0}$, and $\iota_{0}{ }^{*} \theta=$ identity, for any inclusion $\iota_{0}$ of $\mathbf{R}_{k, 2}$ as a fibre in $G_{k, 2}\left(\mathbf{C}_{n, q}\right)$.

Proof. We have the following maps:

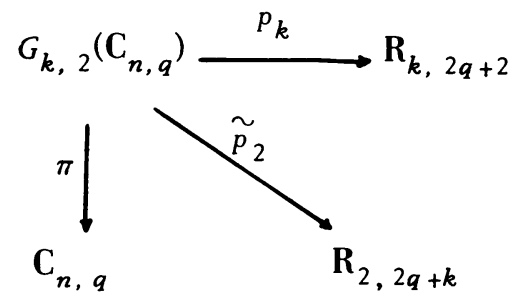

where $p_{k}$ maps any $k$-plane $X$ contained in a complex $n$-plane $\Gamma$ in $\mathrm{C}^{n+q}$ into the same plane in the underlying $\mathbf{R}^{2 n+2 q}$ in $\mathbf{C}^{n+q}$, and $\widetilde{p}_{2}$ maps $X$ into its orthogonal complement in $\Gamma$, considered as a 2 -plane in $\mathbf{R}^{2 n+2 q}$. Define 


$$
\begin{aligned}
\theta\left(\widetilde{\Omega}^{r}\right) & =\epsilon_{r} \tilde{p}_{2}^{*}(\{(r r)\}), \quad \epsilon_{r}=(-1)^{r(r-1) / 2} \text { for } 0 \leq r \leq n-1, \\
\theta(\kappa) & =D K_{0} .
\end{aligned}
$$

Then

$$
\begin{aligned}
\iota_{0}^{*} \theta\left(\tilde{\Omega}^{r}\right) & =\epsilon_{r} \iota_{0}^{*} \tilde{p}_{2}^{*}(\{(r r)\}) & & \\
& =\left\{\left(2_{r}\right)\right\} & & \text { by Proposition } 4.1, \\
& =\widetilde{\Omega}^{r} & & \text { by Proposition } 4.4,
\end{aligned}
$$

and $\iota_{0}^{*} \theta(\kappa)=\iota_{0}^{*} D K_{0}=\kappa$, as is easily seen. Now we define

$$
\theta\left(\kappa \cup \tilde{\Omega}^{r}\right)=\theta(\kappa) \cup \theta\left(\tilde{\Omega}^{r}\right) \quad \text { for } 0 \leq r \leq n-1 .
$$

By Theorem 4.8 this defines $\theta$ on $H^{*}\left(\mathbf{R}_{k, 2}\right)$, and since $\iota_{0}^{*}$ commutes with cup products, we also have $\iota_{0}^{*} \theta=$ identity.

Corollary 5.2. There is an isomorphism of graded groups

$$
\Phi: H_{*}\left(G_{k, 2}\left(\mathbf{C}_{n, q}\right)\right) \rightarrow H_{*}\left(\mathbf{R}_{k, 2}\right) \otimes H_{*}\left(\mathbf{C}_{n, q}\right)
$$

defined by $\Phi(z)=\Sigma_{a} b_{a} \otimes \pi_{*}\left(\theta\left(b_{a}^{*}\right) \cap z\right)$, where $\left\{b_{a}\right\}$ is a basis of $H_{*}\left(\mathbf{R}_{k, 2}\right)$ and $\left\{b_{a}^{*}\right\}$ is the dual basis of $H^{*}\left(\mathbf{R}_{k, 2}\right)$.

Proof. Proposition 5.1 asserts that the bundle $\pi$ has a cohomological extension of the fibre. We know that $H^{*}\left(\mathbf{R}_{k, 2}\right)$ is free, so by the Leray-Hirsch theorem [10] we have the isomorphism $\Phi$.

By Corollary 5.2, we see that to find a basis of $H_{k}\left(G_{k, 2}\left(\mathbf{C}_{n, q}\right)\right)$, we need only find, for each Schubert cycle $Z_{\omega}$ (corresponding to the Schubert symbol $\omega$ ) of $\mathbf{C}_{n, q}$ of degree $(n-1-r), 0 \leq r<n-1$, a k-cycle $Y(\omega)$ of $G_{k, 2}\left(\mathbf{C}_{n, q}\right)$ such that $\pi_{*}\left(\theta\left(\tilde{\Omega}^{r}\right) \cap[Y(\omega)]\right)=\left[\omega^{C}\right]$. These $[Y(\omega)]$, together with $\iota_{0 *} z_{1}$ and $\iota_{0 *} z_{2}$, will then form a basis of $H_{k}\left(G_{k, 2}\left(\mathbf{C}_{n, q}\right)\right)$.

Take a Schubert cycle $Z_{\omega}$ of $\mathrm{C}_{n, q}$ of degree $(n-1-r), 0 \leq r<n-1$. We will find a cycle $Y(\omega)$ in the form of a product bundle over $Z_{\omega}$ with dimension $2 r$ in the fibre. Note that the symbol $\omega=(0 \ldots 0 * \ldots *)$ contains at least $(r+1)$ zeros, and contains at least $(r+2)$ zeros if $\omega \neq(0 \ldots 01 \ldots 1)$. This means that each complex $n$-plane in $Z_{\omega}$ contains a fixed set of orthonormal complex vectors $\sqrt{-1} e_{1}, \cdots, \sqrt{-1} e_{r+1}$, and even contains $\sqrt{-1} e_{r+2}$ if $\omega \neq(0 \ldots 01 \cdots 1)$, when a complex Euclidean metric is given on $\mathbf{C}^{n+q}$. Take any complex $n$-plane $\Gamma$ in $Z_{\omega}$. If $\omega \neq(0 \ldots 01 \cdots 1)$, take the $k$-planes in $\Gamma$ containing the $(2 n-2-r)$-dimensional linear subspace normal to $\sqrt{-1} e_{1}$, $\cdots, \sqrt{-1} e_{r+2}$ in $\Gamma$ with respect to the real inner product induced on $\Gamma$ from $\mathrm{C}^{n+q}$. This is equivalent to taking a Schubert cycle $(2 \ldots 2)$ ( $r$ factors $)$ in the 
fibre over $\Gamma$. Let $Y(\omega)$ be the union of these as $\Gamma$. runs through $Z_{\omega}$. If $\omega=$ $(0 \ldots 01 \ldots 1)$ with $r$ ones, we take $\sqrt{-1} e_{1}, \ldots, \sqrt{-1} e_{r+1}, e_{1}$ and define $Y(\omega)$ similarly. Clearly $Y(\omega)$ is a product bundle over $Z_{\omega}$ with fibre $R_{r, 2}$, and can be taken as a cycle in $G_{k, 2}\left(C_{n, q}\right)$. Let $\left\{Y_{1}, Y_{2}\right\}$ be the basis of $H_{k}\left(\mathbf{R}_{k, 2}\right)$ dual to $\left\{\widetilde{\Omega}^{n-1}, \kappa\right\}$ and let $Y_{j}(0)=\iota_{0 *} Y_{j}, j=1,2$, for an inclusion $\iota_{0}$ of $\mathbf{R}_{k, 2}$ as a fibre in $G_{k, 2}\left(\mathrm{C}_{n, q}\right)$.

Lemma 5.3. Let $\theta$ be as in Proposition 5.1. Then

$$
\pi_{*}\left(\theta\left(\tilde{\Omega}^{s}\right) \cap[Y(\omega)]\right)= \begin{cases}2\left[\omega^{C}\right] & \text { if } s=r, \\ 0 & \text { if } s \neq r\end{cases}
$$

for $\operatorname{deg} \omega=n-r-1,0 \leq r<n-1$. Then

Proof. Let $i: Y(\omega) \rightarrow G_{k, 2}\left(C_{n, q}\right)$ be the inclusion map, and let $\pi^{\prime}=\pi \circ i$.

$$
\begin{aligned}
\pi_{*}\left(\theta\left(\tilde{\Omega}^{s}\right)\right. & \cap[Y(\omega)])=\pi_{*}\left(\theta\left(\tilde{\Omega}^{s}\right) \cap i_{*} Y(\omega)\right) \\
& =\pi_{*} i_{*}\left(i^{*} \theta\left(\tilde{\Omega}^{s}\right) \cap Y(\omega)\right)=\pi_{*}^{\prime}\left(i^{*} \tilde{p}_{2}^{*}\left(\epsilon_{s}\{(s s)\}\right) \cap Y(\omega)\right) \\
& =\pi_{*}^{\prime}\left(\tilde{\Omega}^{s} \times 1 \cap Y(\omega)\right) \\
& = \begin{cases}2\left[\omega^{C}\right] & \text { if } s=r, \\
0 & \text { if } s \neq r .\end{cases}
\end{aligned}
$$

Lemma 5.4. As in Lemma 5.3, we have

$$
\begin{aligned}
\pi_{*}(\theta(\kappa) \cap[Y(\omega)]) & =\left[K_{0}\right] \circ[Y(\omega)] \\
& = \begin{cases}(-1)^{n-r-1} & \text { if } \omega=(\underbrace{1 \ldots 1}_{n-r-1}), \\
0 & \text { if } \omega \neq(1 \ldots 1) .\end{cases}
\end{aligned}
$$

Proof. For $\omega \neq(1 \cdots 1)$, clearly $K_{0} \cap Y(\omega)=\varnothing$. Now consider $\omega=$ $(1 \ldots 1)$ with $(n-r-1)$ ones. Take a Euclidean inner product in $\mathbf{C}^{n+q}$ and - an orthonormal basis $\left\{e_{1}, \cdots, e_{n+q}\right\}$. For any complex $n$-plane $\Gamma^{\prime} \in Z_{\omega}$, let $g_{\Gamma}$ be the complex orthogonal projection of $e_{r+2}$ on $\Gamma$. Then $Y(\omega)$ can be deformed into the subbundle $Y_{g}(\omega)$ of $G_{k, 2}\left(C_{n, q}\right)$ defined by using $\left\{\sqrt{-1} e_{1}\right.$, $\left.\cdots, \sqrt{-1} e_{r+1}, e_{1}+g\right\}$ instead of $\left\{\sqrt{-1} e_{1}, \cdots, \sqrt{-1} e_{r+1}, e_{1}\right\}$. Then $K_{0} \cap Y_{g}(\omega)$ is a singleton set consisting of the complex $(n-1)$-plane perpendicular to $e_{1}$ in the complex $n$-plane spanned by $e_{1}, \ldots, \hat{e}_{r+2}, \cdots$, $e_{n+1}$. One can check the sign of the intersection and verify it to be $(-1)^{n-r-1}$.

Proposition 5.5. Under the isomorphism

$$
\Phi: H_{*}\left(G_{k, 2}\left(\mathbf{C}_{n, q}\right)\right) \rightarrow H_{*}\left(\mathbf{R}_{k, 2}\right) \otimes H_{*}\left(\mathbf{C}_{n, q}\right)
$$


of Corollary 5.2, we have, for any Scbubert symbol $\omega$ of $\mathrm{C}_{n, q}$ of degree $n-r-1,0 \leq r<n-1$,

$$
\begin{aligned}
& \Phi[Y(\omega)]= \begin{cases}{\left[\left(2_{r}\right)\right] \otimes\left[\omega^{c}\right]+(-1)^{n-r-1}\left[Y_{2}\right] \otimes 1} & \text { if } \omega=(1 \cdots 1), \\
{\left[\left(2_{r}\right)\right] \otimes\left[\omega^{c}\right]} & \text { if } \omega \neq(1 \cdots 1),\end{cases} \\
& \Phi\left[Y_{j}(0)\right]=\left[Y_{j}\right] \otimes 1 \quad \text { for } j=1,2 .
\end{aligned}
$$

Proof. Note that $\left\{\tilde{\Omega}^{r}, \widetilde{\Omega}^{r} \cup \kappa\right\}, r=0, \cdots, n-1$, forms a basis of $H *\left(\mathbf{R}_{k, 2}\right)$, and the dual basis consists of $1 / 2\left[\left(2_{r}\right)\right]$ for $0 \leq r<n-1$, together with $Y_{1}, Y_{2}$ and homology classes of higher dimensions which we are not interested in. Therefore,

$$
\begin{aligned}
\Phi[Y(\omega)]= & \sum_{s=0}^{n-2} 1 / 2\left[\left(2_{s}\right)\right] \otimes \pi_{*}\left(\theta\left(\widetilde{\Omega}^{s}\right) \cap[Y(\omega)]\right) \\
& +\left[Y_{1}\right] \otimes \pi_{*}\left(\theta\left(\widetilde{\Omega}^{n-1}\right) \cap[Y(\omega)]\right)+\left[Y_{2}\right] \otimes \pi_{*}(\theta(\kappa) \cap[Y(\omega)]) .
\end{aligned}
$$

The first formula then follows from Lemma 5.3 and Lemma 5.4. The second formula is similarly proved.

Corollary 5.6. The homology classes $[Y(\omega)], 1 \leq \operatorname{deg} \omega \leq n-1$, together with $\left[Y_{1}(0)\right]$ and $\left[Y_{2}(0)\right]$, form a linear basis of $H_{k}\left(G_{k, 2}\left(\mathbf{C}_{n, q}\right)\right) \otimes \mathbf{R}$.

Let $\Omega_{0}=p_{k}^{*}\{(1 \cdots 1)\} \in H^{k}\left(G_{k, 2}\left(\mathrm{C}_{n, q}\right)\right)$ be the Euler class of the natural $k$-plane bundle over $G_{k, 2}\left(C_{n, q}\right)$. We continue to assume $k=2 n-2$.

Lemma 5.7.

$$
\begin{array}{ll}
\left\langle\Omega_{0},\left[Y_{j}(0)\right]\right\rangle=\left\langle 2 \kappa-\widetilde{\Omega}^{n-1},\left[Y_{j}\right]\right\rangle & \text { for } j=1,2, \\
\left\langle\Omega_{0},[Y(\omega)]\right\rangle=0 & \text { if } \operatorname{deg} \omega>0 .
\end{array}
$$

Proof. The first formula follows from Theorem 4.8 and the observation that $\iota_{0}^{*} \Omega_{0}=\Omega \in H^{k}\left(\mathbf{R}_{k, 2}\right)$. For $\operatorname{deg} \omega>0$ and

$$
\omega \neq(\underbrace{1 \ldots 1}_{n-1}),
$$

all the $k$-planes in $Y(\omega)$ contain the vector $e_{2}$, that is, the $k$-plane bundle restricted over $Y(\omega)$ has a nonzero section, and so $\left\langle\Omega_{0},[Y(\omega)]\right\rangle=0$. For

$$
\omega=(\underbrace{1 \ldots 1}_{n-1})
$$

$Y(\omega)$ consists of two disjoint components, and the $k$-plane bundles over them are oppositely oriented, so that the values of $\Omega_{0}$ on them cancel with each other. 
Lemma 5.8. For $\operatorname{deg} \omega>0$,

$$
\left\langle\theta\left(\tilde{\Omega}^{r}\right) \cap \pi^{*}\left(c_{n-r-1}\right),[Y(\omega)]\right\rangle= \begin{cases}2(-1)^{n-r-1} & \text { if } \omega=\underbrace{(\ldots 1}_{n-r-1}) \\ 0 & \text { otherwise, }\end{cases}
$$

where $c_{n-r-1}=(-1)^{n-r-1}\left\{(1 \ldots 1)^{\mathrm{C}}\right\} \in H^{2(n-r-1)}\left(\mathbf{C}_{n, q}\right)$.

Proof: The left-hand side equals $\pi^{*}\left(c_{n-r-1} \cap \pi_{*}\left(\theta\left(\tilde{\Omega}^{r}\right) \cap[Y(\omega)]\right)\right)$. Apply Lemma 5.3.

Proposition 5.9. In $H^{k}\left(G_{k, 2}\left(\mathrm{C}_{n, q}\right)\right)$,

$$
\Omega_{0}+\sum_{r=0}^{n-1} \theta\left(\tilde{\Omega}^{r}\right) \cup \pi^{*}\left(c_{n-r-1}\right)=2 D K_{0} .
$$

Proof. We need only evaluate both sides on the homology basis of Corollary 5.6 and verify that they are equal. The necessary formulas are contained in Proposition 5.1 and Lemmas 5.4, 5.7 and 5.8.

Theorem 5.10. Let $\xi \oplus \xi^{\prime}=\eta$ be a decomposition of a complex n-plane bundle $\eta$ over $M$ into an oriented $(2 n-2)$-plane bundle $\xi$ and a 2-plane bundle $\xi^{\prime}$. Let $t: M \rightarrow G_{k, 2}(\eta)$ be the section corresponding to the decomposition. Then

$$
\Omega(\xi)+\sum_{r=0}^{n-1} \Omega\left(\xi^{\prime}\right)^{r} \cup c_{n-r-1}(\eta)=2 t^{*} D K_{\eta}
$$

Proof. We have the commutative diagram

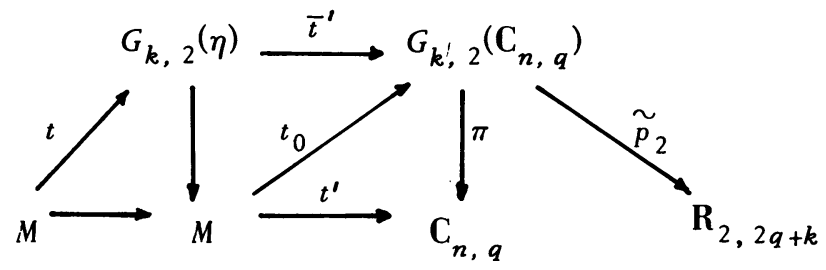

where $t^{\prime}$ induces $\eta$, and $t_{0}=\bar{t}^{\prime} \circ t$. Clearly $\bar{t}^{\prime}\left(K_{\eta}\right)=K_{0}$, and $\xi$, $\xi^{\prime}$ are induced by $t_{0}$ from the natural $k$-plane and 2-plane bundles over $G_{k, 2}\left(\mathrm{C}_{n, q}\right)$. Therefore

$$
\begin{aligned}
t_{0}^{*} \Omega_{0} & =\Omega(\xi), \\
t_{0}^{*} \theta\left(\tilde{\Omega}^{r}\right) & =t_{0}^{*} \tilde{p}_{2}^{*}\left(\epsilon_{r}\{(r r)\}\right) \quad(\text { see Proposition 5.1) } \\
& =\Omega\left(\xi^{\prime}\right)^{r}, \\
t_{0}^{*} \pi^{*} c_{n-r-1} & =t^{\prime *} c_{n-r-1}=c_{n-r-1}(\eta), \\
t_{0}^{*} D_{0} K_{0} & =t^{*} D_{\eta} K_{\eta},
\end{aligned}
$$


where $D_{0}, D_{\eta}$ are the Poincaré dualities in $G_{k, 2}\left(C_{n, q}\right)$ and $G_{k, 2}(\eta)$ respectively. The theorem then follows from Proposition 5.9.

\section{Applications.}

I. Duality theorems for Stiefel-Whitney classes and Chern classes. If we reduce the coefficients mod 2 , Theorem 5.10 becomes

$$
\Omega(\xi)+\sum_{r=0}^{n-1} \Omega\left(\xi^{\prime}\right)^{r} \cup c_{n-r-1}(\eta) \equiv 0 \bmod 2
$$

or

$$
W_{2 n-2}(\xi)+\sum_{r=0}^{n-1} W_{2}\left(\xi^{\prime}\right)^{r} \cup W_{2 n-2 r-2}(\eta)=0 .
$$

This is a particular case of the Whitney duality theorem for $\xi \oplus \xi^{\prime}=\eta$ with $W_{1}\left(\xi^{\prime}\right)=0$ (orientability).

Suppose $\xi, \xi^{\prime}$ are complex vector bundles and we replace $\xi$ by $-\xi$ and $\xi^{\prime}$ by $-\xi^{\prime}$ in Theorem 5.10, then the Euler classes change sign, and $t^{*} D K_{\eta}=0$. Therefore

$$
-\Omega(\xi)+\sum_{r=0}^{n-1}(-1)^{r} \Omega\left(\xi^{\prime}\right)^{r} \cup c_{n-r-1}(\eta)=0
$$

or

$$
c_{n-1}(\xi)=\sum_{r=0}^{n-1}(-1)^{r} c_{1}\left(\xi^{\prime}\right)^{r} \cup c_{n-r-1}(\eta)
$$

which is a particular case of the duality theorem for Chern classes.

Remark. If $\xi \oplus \xi^{\prime}=\eta$ is a decomposition of a complex $n$-plane bundle into two complex bundles, where $\xi^{\prime}$ is a complex line bundle, the duality theorem for Chern classes implies that $\Omega(\xi)-\sum_{r=0}^{n-1}(-1)^{r} \Omega\left(\xi^{\prime}\right)^{r} \cup c_{n-r-1}(\eta)=0$, whereas if $\xi^{\prime}$ is just an orientable 2-plane bundle, the duality theorem for Stiefel-Whitney classes implies that $\Omega(\xi)-\sum_{r=0}^{n-1}(-1)^{r} \Omega\left(\xi^{\prime}\right)^{r} \cup c_{n-r-1}(\eta)$ is an even class. Theorem 5.10 describes this even class explicitly in terms of the real-complex interaction.

II. Index of RC-singularity. We can define the index of RC-singularity at an RC-singular point of an immersion $\iota: M^{(2 n-2)} \rightarrow N_{(n)}$, or, more generally, of an oriented $(2 n-2)$-plane subbundle of a complex $n$-plane bundle over a compact orientable manifold of dimension $(2 n-2)$, in the same way as we did in $\$ 4$. As examples of applications of Theorem 5.10, we prove the following two theorems:

Theorem 5.11. Suppose that a compact $(n-1)$-dimensional complex manifold 
$M$ immersed in an $n$-dimensional complex manifold $N$ is slightly deformed so that the RC-singular points are all isolated. Then the sum of the indices of RC-singularity is equal to $c_{n-1}(N)+\widetilde{\Omega}^{2} \cup c_{n-3}(N)+\cdots$ evaluated on $M$, where $\widetilde{\Omega}$ denotes the Euler class of the normal bundle.

Proof. Let $\xi, \xi^{\prime}$ be the tangent and normal bundles of $M$ in $N$. The duality theorem for Chern classes yields

$$
c_{n-1}(\xi)+\sum_{r=0}^{n-1}(-1)^{r} c_{1}\left(\xi^{\prime}\right)^{r} \cup c_{n-r-1}(N),
$$

whereas Theorem 5.10 gives

$$
c_{n-1}(\xi)+\sum_{r=0}^{n-1} c_{1}\left(\xi^{\prime}\right)^{r} \cup c_{n-r-1}(N)=2 t^{*} D K_{\eta} .
$$

Addition gives $t^{*} D K_{\eta}=c_{n-1}(N)+\widetilde{\Omega}^{2} \cup c_{n-3}(N)+\cdots$.

Theorem 5.12. If an oriented $2 m$-plane bundle over $S^{2 m}, m>1$ (the sphere of dimension $2 m$ ), can be embedded in a trivial $(2 m+2)$-plane bundle, then its Euler class is even, and can be interpreted as twice the sum of indices of $\mathbf{R C}$-singularity over the set of RC-singular points where the tangent planes are complex oriented, when a suitable complex structure is introduced on the trivial bundle.

Proof. With suitable deformation we can assume that the RC-singular points are all isolated. Then $\left\langle t^{*} D K, S^{2 m}\right\rangle$ gives the sum of indices of RC-singularity in the statement of the theorem. Because $S^{2 m}$ has no cohomology classes of dimension 2, we see that Theorem 5.10 becomes $\Omega=2 t^{*} D K$. The theorem follows.

6. Immersions of general codimensions. We have seen in Theorem 2.3 that, for a generic immersion of $M^{(k)}$ in $\mathrm{C}^{n}$, the dimension of the set of RC-singular points is equal to

$$
k-2(k-n+1)=2 n-k-2 .
$$

For $k=2 n-2$, this implies that the RC-singular points are isolated, and we have the results of $\$ \$ 4$ and 5 . For higher codimensions, this dimension will be positive, and we expect to find subsets of the set of RC-singular points which carry some nonzero cohomology classes of $M$. We will in fact show that, under some dimensional restrictions, all the dual Pontrjagin classes and the Euler class of $M$ are carried by certain subsets of the set of $\mathbf{R C}$-singular points. First we need to find a basis for the rational homology groups of $\mathbf{R}_{k, k^{\prime}}$ in the. appropriate dimensions. Let $\mathbf{Q}$. be the field of rational numbers. 
Lemma 6.1. (i) For $4 r<\min \left(k, k^{\prime}\right), H_{4 r}\left(\mathbf{R}_{k, k^{\prime}} ; \mathbf{Q}\right)$ bas as basis the bomology classes represented by the Schubert symbols each of whose nonzero entries is even and occurs an even number of times. For $4 r=k<k^{\prime}$, there is one more bas is member $\left[(1 \cdots 1)^{+}-(1 \cdots 1)^{-}\right]$; for $4 r=k^{\prime}<k$, there is one more basis member $\left[(4 r)^{+}-(4 r)^{-}\right]$; and for $4 r=k=k^{\prime}$, all these classes together form a basis.

Proof. Pontrjagin has already computed a free basis of $H^{q}\left(\mathbf{R}_{k, k^{\prime}}\right)$ for $q<k^{\prime}$ (Theorem 1 of [8]). For the case $4 r<\min \left(k, k^{\prime}\right)$ this implies that $H^{4 r}\left(\mathbf{R}_{k, k^{\prime}} ; \mathbf{Q}\right)$ has as basis the cohomology classes $\left\{\left(a_{1}, \ldots, a_{k}\right)^{+}+\right.$ $\left.\left(a_{1}, \cdots, a_{k}\right)^{-}\right\}$, where each nonzero entry is even and occurs an even number of times. The corresponding homology classes are $\left[\left(a_{1}, \ldots, a_{k}\right)^{+}+\left(a_{1}, \ldots, a_{k}\right)^{-}\right]$, and their Kronecker products are either 2 or 0 depending on whether the Schubert symbols are the same or not. Therefore, by the Kronecker pairing these homology classes form a basis of $H_{4 r}\left(\mathbf{R}_{k, k^{\prime}} ; \mathbf{Q}\right)$.

For the case $4 r=k<k^{\prime}$, there is just one more basis element for $H^{k}\left(\mathbf{R}_{k, k^{\prime}} ; \mathbf{Q}\right)$ than $H^{k}\left(\mathbf{R}_{k+1, k^{\prime},} ; \mathbf{Q}\right)$, namely the class $\{(1 \cdots 1)\}$. On the other hand, the relation between $H^{k}\left(\mathbf{R}_{k, k^{\prime}}\right)$ and $H^{k^{\prime}}\left(\mathbf{R}_{k, k^{\prime}+1}\right)$ has been studied by Lashof and Smale (Theorem 4.2 of [7]), and taking rational coefficients we see that $H^{k^{\prime}}\left(\mathbf{R}_{k, k^{\prime}}\right)$ is generated by $H^{k^{\prime}}\left(\mathbf{R}_{k, k^{\prime}+1}\right)$ and the class $\left\{\left(k^{\prime}\right)\right\}$. Therefore similar arguments as before can be applied.

We now construct the cycles $L_{r}(k, k)$ which will carry the dual Pontrjagin classes of $\mathbf{R}_{k, k}$. Fix a complex structure in $\mathbf{R}^{2 k}$ compatible with the orientation. There is a natural complex $(k-1)$-plane bundle over $\mathbf{C}_{1, k-1}$, and the total space of the associated bundle with fibre $\mathbf{R}_{k-2, k}$ is denoted by $G_{k-2, k}\left(\mathbf{C}_{1, k-1}\right)$. There is a natural map

$$
\psi: G_{k-2, k}\left(\mathbf{C}_{1, k-1}\right) \rightarrow \mathbf{R}_{k, k}
$$

which sends a $(k-2)$-plane $Y$ in the fibre over $X \in \mathbf{C}_{1, k-1}$ into the oriented $k$-plane $X+Y$ in $\mathbf{R}^{2 k}$. Let $K_{k, k}$ be the subset of $\mathbf{R}_{k, k}$ consisting of the $k$-planes which contain nontrivial complex subspaces. If $t: M \rightarrow \mathbf{R}_{k, k}$ is the Gauss map of an immersion of $M$ into $C^{k}$, it is clear that $t^{-1}\left(K_{k, k}\right)$ is the set of RC-singular points. We note that $\psi$ is an immersion of $G_{k-2, k}\left(\mathrm{C}_{1, k-1}\right)$ into $\mathbf{R}_{k, k}$ whose image is precisely $K_{k, k}$.

Definition 6.2. Let $L_{r}(k, k), 4 \leq 4 r \leq k$, be the image under $\psi$ of the subbundle of $G_{k-2, k}\left(\mathrm{C}_{1, k-1}\right)$ over the Schubert cycle $(k-2 r)^{\mathrm{C}}$ of the base, and let the orientation be $(-1)^{k(k-1) / 2}$ times that induced from the bundle structure.

Proposition 6.3. In $H^{4 r}\left(\mathbf{R}_{k, k}\right), D\left[L_{r}(k, k)\right]=\bar{P}_{r} \bmod$ torsion. 
This proposition, as well as Propositions 6.5 and 6.9, can be proved by finding the intersections of the cycle under study with the representative cycles of a homology basis (defined with respect to some suitable coordinate system) as indicated by Lemma 6.1. The method is similar to that of Lemma 4.7, although the details are quite different, and by no means trivial. They will be omitted from this paper, and reference is made to the Ph.D. dissertation of the author at the University of California, Berkeley, 1971.

For the case $k=n+1$, we have a bundle $G_{k-4, k-2}\left(\mathrm{C}_{2, k-3}\right) \rightarrow \mathrm{C}_{2, k-3}$ with fibre $\mathbf{R}_{k-4, k-2}$ and a natural immersion

$$
\psi: G_{k-4, k-2}\left(\mathbf{C}_{2, k-3}\right) \rightarrow \mathbf{R}_{k, k-2}
$$

whose image is $K_{k, k-2}$, the set of $k$-planes in $\mathbf{C}^{k-1}=\mathbf{R}^{2 k-2}$ which contain a two-dimensional complex subspace.

Definition 6.4. Let $L_{r}(k, k-2), 4 \leq 4 r \leq k$, be the image under $\psi$ of the subbundle of $G_{k-4, k-2}\left(C_{2, k-3}\right)$ over the Schubert cycle $(k-2 r-1, k-3)^{\mathrm{C}}$ of the base, and let the orientation be $(-1)^{k-1}$ times that induced from the bundle structure.

Proposition 6.5. In $H^{4 r}\left(\mathbf{R}_{k, k-2}\right), 4 \leq 4 r \leq k$, we have

$$
D\left[L_{r}(k, k-2]=\bar{P}_{r} \bmod \right. \text { torsion. }
$$

As consequences of Propositions 6.3 and 6.5, we have

Theorem 6.6. Let $t$ be the Gauss map of an immersion of $M^{(k)}$ in $\mathbf{C}^{n}$, where $k=n$ or $n+1$. Then

$$
t^{*} D\left[L_{r}\right]=\bar{P}_{r}(M) \bmod \text { torsion }
$$

for $4 \leq 4 r \leq k$, where $L_{r}=L_{r}(k, k)$ if $k=n$ and $L_{r}=L_{r}(k, k-2)$ if $k=n+1$.

Corollary 6.7. If $\mathrm{M}^{(k)}$ can be immersed in $\mathbf{C}^{k}$ or $\mathbf{C}^{k-1}$ without RC-singular points, then all Pontrjagin classes of $M$ except $P_{0}$ are zero $\bmod$ torsion.

The first part of Corollary 6.7 has been proved by Wells [15]. Theorem 6.6 therefore generalizes Wells's result.

We now show how the Euler class of $M$ is carried by the set of RC-singular points. Let $k$ be even and $n \leq k<2 n-2$. For convenience, let $p=2 n-k-2$ and $q=k-n+1$. Then, as before, we have a bundle $G_{p, p+2}\left(\mathbf{C}_{q, p+1}\right)$ over $\mathrm{C}_{q, p+1}$ with fibre $\mathbf{R}_{p, p+2}$, and a natural immersion

$$
\psi: G_{p, p+2}\left(\mathbf{C}_{q, p+1}\right) \rightarrow \mathbf{R}_{k, 2 n-k}
$$

whose image is $K_{k, 2 n-k}$, the set of $k$-planes in $\mathrm{C}^{n}=\mathrm{R}^{2 n}$ which contain a 
$(k-n+1)$-dimensional complex subspace. We fix an oriented orthonormal basis $f_{1}, \cdots, f_{2 n}$ of $\mathbf{R}^{2 n}$ and a complex basis $g_{1}, \cdots, g_{n}$ of $\mathbf{C}^{n}$ such that

$$
\sqrt{-1} g_{j}=\sqrt{-1} f_{j}=f_{2 n+1-j} \text { for } j=1, \cdots, n \text {. }
$$

Take Schubert chains of $\mathrm{C}_{q, p+1}$ relative to $\left\{g_{j}\right\}$. No complex $q$-plane $X \epsilon$ $\left(a_{1}, \cdots, a_{q}\right)_{0}^{\mathrm{C}}$ with $a_{1}>0$ contains $g_{1}$, and so its normal plane $X^{\perp}$ is not contained in the linear space $\mathbf{R}_{*}^{2 n-1}$ spanned by $f_{2}, \cdots, f_{2 n}$. Consequently $\operatorname{dim}\left(X^{\perp} \cap \mathbf{R}_{*}^{2 n-1}\right)=2 p+1$. The Grassmannian of oriented $p$-planes in $X^{\perp} \cap \mathbf{R}_{*}^{2 n-1}$ is the Schubert cycle $\left[(p+1, \cdots, p+1)^{+}-(p+1, \cdots, p+1)^{-}\right]$ in $X^{\perp}$, and is naturally oriented independently of the orientation of $X^{\perp} \cap R_{*}^{2 n-1}$ chosen to define it.

Definition 6.8. Let $L(k, 2 n-k)$ be the image under $\psi$ of the subset of $G_{p, p+2}\left(\mathbf{C}_{q, p+1}\right)$ consisting of (i) all p-planes in $X^{\perp} \cap \mathbf{R}_{*}^{2 n-1}$ for $X \epsilon$ $\left(a_{1}, \cdots, a_{q}\right)_{0}^{\mathrm{C}} \subset \mathbf{C}_{q, p+1}$ with $a_{1}>0$, and (ii) the whole fibres over each $X \in\left(0, \ldots, a_{q}\right) \mathrm{C} \subset \mathrm{C}_{q, p+1}$. In the interior of the subset defined by (i), let the orientation be $(-1)^{1 / 2 k n}$ times that induced from the orientation of the Schubert cycle $[(p+1, \cdots, p+1)]$.

We note that the dimension of the part (i) is

$$
p(p+1)+2 q(p+1)=k(2 n-k-1),
$$

while the dimension of the part (ii) is at most

$$
p(p+2)+\operatorname{dim} \mathbf{C}_{k-n, p+1}=k(2 n-k-1)-(2 n-k)<k(2 n-k-1)-2 .
$$

Consequently, the part (ii) does not affect the dimension, and $L$ is a pseudomanifold representing a cycle in $\mathbf{R}_{k, 2 n-k}$.

Proposition 6.9. In $H^{k}\left(\mathbf{R}_{k, 2 n-k}\right)$, we have, mod torsion,

$$
D[L(k, 2 n-k)]= \begin{cases}\Omega+\epsilon \widetilde{\Omega} & \text { with } \epsilon= \pm 1 \text { if } k=n, \\ \Omega & \text { if } k>n .\end{cases}
$$

Theorem 6.10. For any immersion of $M^{(k)}$ into $\mathrm{C}^{n}$ with Gauss map t,

$$
t^{*} D[L(k, 2 n-k)]= \begin{cases}\Omega(M)+\epsilon \widetilde{\Omega}(\iota) & \text { with } \epsilon= \pm 1 \text { if } k=n, \\ \Omega(M) & \text { if } k>n .\end{cases}
$$

In particular, for an embedding of $M^{(k)}$ into $\mathbf{C}^{n}, n \leq k<2 n-2$,

$$
t^{*} D[L(k, 2 n-k)]=\Omega(M) \text {. }
$$

Proof. The first statement follows from Proposition 6.9, and the second follows from the fact that the normal Euler class of an embedding of a compact orientable manifold in Euclidean space is zero. 
Again this generalizes a result of Wells [15], which asserts that if $M^{(k)}$ can be embedded in $\mathbf{C}^{n}(k \geq n)$ without $\mathbf{R C}$-singular points, then the Euler characteristic of $M$ must be zero. (We note that for $k$ odd the conclusion is automatic by Poincaré duality.)

7. The RC-ring of an embedding. In this section all homology and cohomology are taken with coeffients in a field of characteristic zero. We will express the results of $\$ \$ 4$ and 6 in a neater form.

Lemma 7.1. Let $\iota: M^{(k)} \rightarrow \mathrm{C}^{n}$ be an embedding. Then the induced cobomology bomomorphism of the Gauss map $t: M \rightarrow \mathbf{R}_{k, 2 n-k}$ bas image equal to the characteristic ring of $M$.

Proof. Lashof and Smale [7] have shown that for $r<k, H^{r}\left(\mathbf{R}_{k, 2 n-k}\right)$ is generated as a ring by $\widetilde{\Omega}$ and the Pontrjagin classes. Now $H^{k}\left(\mathbf{R}_{k, 2 n-k}\right)$ is generated as a vector space by $\Omega$ and $H^{k}\left(\mathbf{R}_{k+1,2 n-k}\right)$, and to the latter the same theorem applies. Therefore for $r \leq k, H^{r}\left(\mathbf{R}_{k, 2 n-k}\right)$ is generated as a ring by $\Omega, \widetilde{\Omega}$ and the Pontrjagin classes. Now $t^{*}$ maps $\widetilde{\Omega}$ into zero. So the image of $t^{*}$ is generated by the Euler class and all the Pontrjagin classes of $M$, and is therefore equal to the characteristic ring of $M$.

Definition 7.2. The RC-ring of an embedding $M^{(k)} \rightarrow \mathrm{C}^{n}$ with Gauss map $t: M \rightarrow \mathbf{R}_{k, 2 n-k}$ is the image under $t^{*}$ of the set of all cohomology classes of $\mathbf{R}_{k, 2 n-k}$ whose dual homology classes have representative cycles which are contained in $K_{k, 2 n-k}$.

Proposition 7.3. The RC-ring of an embedding $\iota: M^{(k)} \rightarrow \mathbf{C}^{n}$ is a proper ideal of the characteristic ring of $M$.

Proof. By Lemma 7.1 the RC-ring is a subset of the characteristic ring, and is clearly proper. That it is an ideal follows by transforming cup products of cohomology classes into intersections of representative cycles by Poincaré duality.

Geometrically, the RC-ring of an embedding is the ring of cohomology classes whose dual homology classes have representative cycles contained in the set of RC-singularities of the embedding, prcvided that the embedding is generic enough. It would therefore be of interest to find the size of the RC-ring. Theorems 4.8, 6.6, and 6.10 can now be summarized in the following form:

Theorem 7.4. The RC-ring of an embedding $M^{(k)} \rightarrow \mathrm{C}^{n}$ with $k=n, n+1$ or $2 n-2$ is equal to the maximal ideal of the characteristic ring of $M$ (that is, the set of all the nonunits of the characteristic ring).

There arises naturally the question whether the RC-ring can be equal to the 
maximal ideal of the characteristic ring if $n+1<k<2 n-2$. The answer is negative, for dimension reasons. In $\$ 2$ we have shown that

$$
\operatorname{dim} \mathbf{R}_{k, 2 n-k}-\operatorname{dim} K_{k, 2 n-k}=2(k-n+1) .
$$

If $4 \leq k<2 n-2$, then the first Pontrjagin class $P_{1}$ occurs in the characteristic ring, but if also $n+1<k$, then the RC-ring does not have any 4-dimensional component, and so cannot be the maximal ideal of the characteristic ring. However, it can be shown that the RC-ring contains all the Pontrjagin classes $P$, with $2(k-n+1) \leq 4 r \leq k$.

In the special case of $k=n+2 \geq 8$, it can be shown that the 8-dimensional component is generated by $P_{2}$, and that $\bar{P}_{2}$ does not occur in the RC-ring. Higher dimensional components become more and more involved.

8. Second order singularities. So far we have considered only RC-singular points of the first order, that is, at which the tangent space contains a complex subspace of dimension one higher than the minimal. It is natural to consider the RC-singular points at which the tangent space contains higher dimensional complex subspaces.

Definition 8.1. A point $x \in M$ is called an RC-singular point of second order of the immersion $\iota: M^{(k)} \rightarrow \mathrm{C}^{n}(k \geq n)$ if

$$
\operatorname{dim}_{C} \iota_{*} T_{x}(M) \cap J \iota_{*} T_{x}(M) \geq k-n+2 .
$$

Clearly the set of RC-singular points of second order is a subset of, and generically of lower dimension than, the set of RC-singular points. Let $K_{k, 2 n-k}^{(2)}$ be the subset of $\mathbf{R}_{k, 2 n-k}$ consisting of the $k$-planes in $\mathbf{C}^{n}$ containing a $(k-n+2)$-dimensional complex subspace. Again there is a bundle $G_{2 n-k-4,2 n-k}\left(\mathrm{C}_{k-n+2,2 n-k-2}\right) \rightarrow \mathrm{C}_{k-n+2,2 n-k-2}$ with fibre $\mathbf{R}_{2 n-k-4,2 n-k}$, and an immersion of $G_{2 n-k-4,2 n-k}\left(\mathbf{C}_{k-n+2,2 n-k-2}\right)$ in $\mathbf{R}_{k, 2 n-k}$ which exhibits $K_{k, 2 n-k}^{(2)}$ as an immersed submanifold of $\mathbf{R}_{k, 2 n-k}$. As an example of the general situation, we have the following proposition.

Proposition 8.2. In $H^{8}\left(\mathbf{R}_{k, k}\right)$ with $k \geq 8$,

$$
D\left[K_{k, k}^{(2)}\right]=P_{1}^{2} \bmod \text { torsion. }
$$

Proof. Note that

$$
\operatorname{dim} K_{k, k}^{(2)}=k(k-4)+4(k-2)=k^{2}-8,
$$

so that $D\left[K_{k, k}^{(2)}\right]$ is of dimension 8 . We know that $H_{8}\left(\mathbf{R}_{k, k} ; \mathbf{Q}\right)$ has a free basis consisting of $[(44)]$ and $[(2222)]$, together with $[(8)]$ and $[(1 \cdots 1)]$ if $k=8$. We can find the intersections of $K_{k, k}^{(2)}$ with these cycles and verify that 


$$
\begin{aligned}
D\left[K_{k, k}^{(2)}\right] & =\{(44)\}+\{(2222)\}=P_{2}+\bar{P}_{2} \\
& =-P_{1} \bar{P}_{1} \bmod \text { torsion, by duality } \\
& =P_{1}^{2} .
\end{aligned}
$$

Theorem 8.3. Let $t: M^{(k)} \rightarrow \mathbf{R}_{k, k}$ be the Gauss map of an immersion $M^{(k)} \rightarrow \mathrm{C}^{k}, k \geq 8$. Then

$$
t^{*} D\left[K_{k, k}^{(2)}\right]=P_{1}(M)^{2} \bmod \text { torsion. }
$$

In particular, we have

Corollary 8.4. If $P_{1}(M)^{2}$ is not torsion, than any immersion of $M^{(k)}$ in $C^{k}$, $k \geq 8$, bas points where the tangent plane contains a complex subspace of complex dimension 2.

\section{BIBLIOGRAPHY}

1. E. Bishop, Differentiable manifolds in complex Euclidean space, Duke Math. J. 32 (1965), 1-21. MR 34 \#369.

2. E. Cartan, Sur la géométrie pseudo-conforme des hypersurfaces de l'espace de deux variables complexes. I, Ann. Mat. 11 (1932), 17-90.

3. S. S. Chern, Topics in differential geometry, The Institute for Advanced Study, Princeton, N. J., 1951 (mimeographed). MR 19, 764.

4. S. S. Chern and E. Spanier, A theorem on orientable surfaces in four-dimensional space, Comment. Math. Helv. 25 (1951), 205-209. MR 13, 492.

5. W. Gysin, Zur Homologie theorie der Abbildungen und Faserungen von Mannigfaltigkeiten, Comment. Math. Helv. 14 (1942), 61-122. MR 3, 317.

6. L. R. Hunt, The envelope of holomorphy of an n-manifold in $\mathrm{C}^{n}$, Boll. Un. Mat. Ital. 4 (1971), 12-35.

7. R. Lashof and S. Smale, On the immersion of manifolds in Euclidean space, Ann. of Math. (2) 68 (1958), 562-583. MR 21 \#2246.

8. L. S. Pontrjagin, Characteristic cycles on differentiable manifolds, Mat. Sb. 21 (63) (1947), 233-284; English transl., Amer. Math. Soc. Transl. (1) 7 (1962), 149-219. MR 9, 243.

9. H. Seifert, Algebraische Approximation von Mannigfaltigkeiten, Math. Z 41 (1936), $1-17$.

10. E. Spanier, Algebraic topology, McGraw-Hill, New 'York, 1966. MR 35 \#1007.

11. N. Tanaka, On the pseudo-conformal geometry of hypersurfaces of the space of $n$ complex variables, J. Math. Soc. Japan 14 (1962), 397-429. MR 26 \#3086.

12. - On generalized graded Lie algebras and geometric structures. I, J. Math. Soc. Japan 19 (1967), 215-254. MR 36 \#4470.

13. R. Thom, Un lemme sur les applications différentiables, Bol. Soc. Mat. Mexicana (2) 1 (1956), 59-71. MR 21 \#910.

14. R. O. Wells, Jr., Holomorphic hulls and holomorphic convexity, Complex Analysis (Proc. Conf. Rice Univ., Houston, Tex., 1967), Rice Univ. Studies 54 (1968), no. 4, 75-84. MR 39 \#3029.

15. - Compact real submanifolds of a complex manifold with nondegenerate holomorphic tangent bundles, Math. Ann. 179 (1969), 123-129. MR 38 \#6104. 
16. R. O. Wells, Jr., Concerning the envelope of holomorphy of a compact differentiable submanifold of a complex manifold, Ann. Scuola Norm. Sup. Pisa (3) 23 (1969), 347361. MR $39 \# 7141$.

17. W. T. Wu, Sur les classes caractéristiques des structures fibrées sphériques, Actualités Sci. Indust., no. 1183 = Publ. Inst. Math. Univ. Strasbourg 11 pp. 5-89, 155156, Hermann, Paris, 1952. MR 14, 1112.

DEPARTMENT OF MATHEMATICS, TULANE UNIVERSITY, NEW ORLEANS, LOUISIANA 70118 